Alma Mater Studiorum - Università di Bologna DEPARTMENT OF ECONOMICS

\title{
GMM estimation of fiscal rules: Monte Carlo experiments and empirical tests
}

\author{
Irene Mammi
}

Quaderni - Working Paper DSE $N^{\circ} 1028$

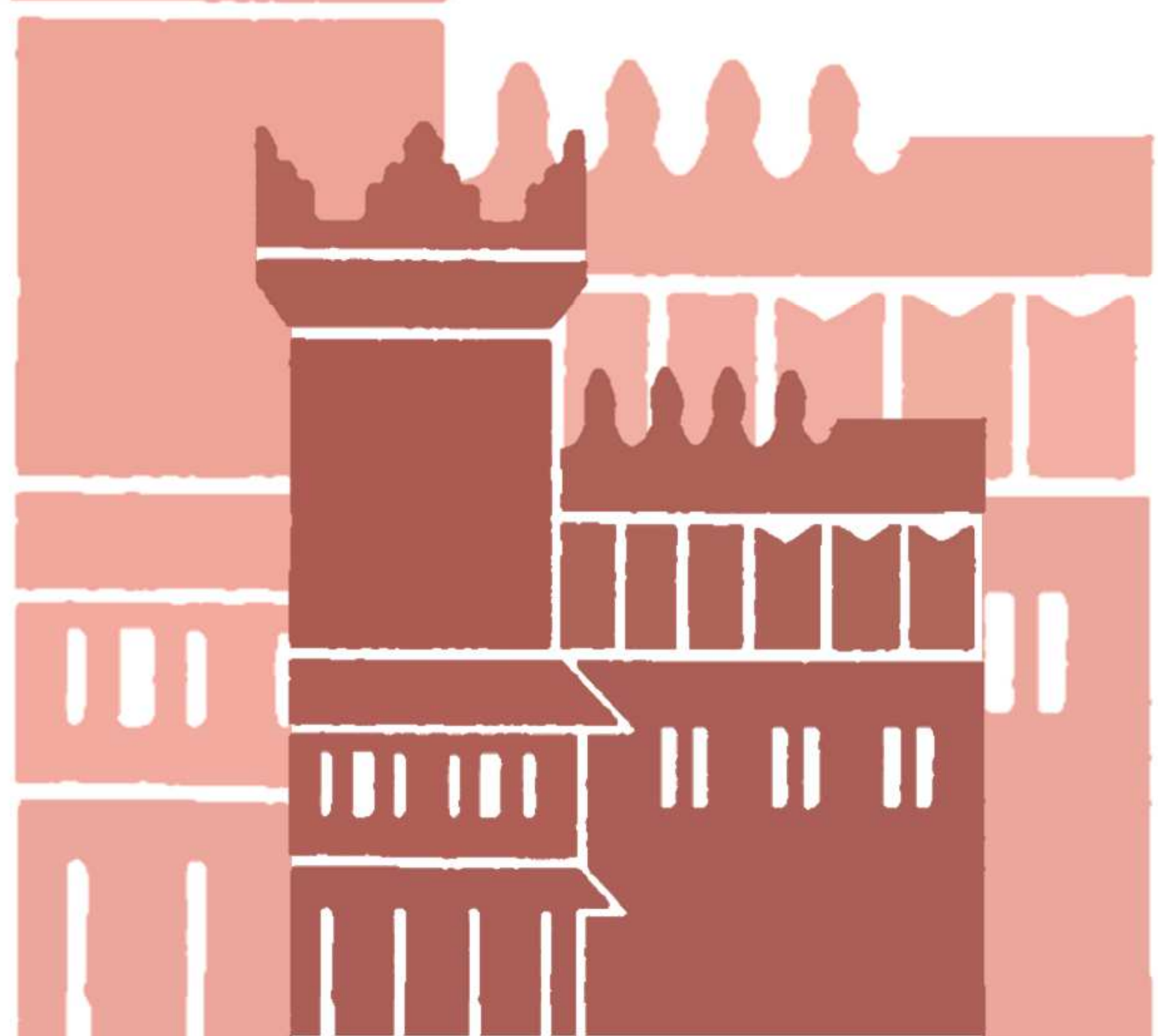




\title{
GMM estimation of fiscal rules: Monte Carlo experiments and empirical tests
}

\author{
Irene Mammi* \\ University of Bologna
}

31 August 2015

\begin{abstract}
This paper focuses on the estimation of fiscal response functions for advanced economies and on the performance of alternative specifications of the Generalized Method of Moments (GMM) estimator for the rule's parameters. We first estimate the parameters on simulated data through Monte Carlo experiments; we then run an empirical test on data for the European Monetary Union (EMU). We estimate both the Cyclicallyadjusted primary balance (CAPB) and the Primary balance (PB) models, and check the robustness of the estimates to different specifications of the GMM estimator and to alternative settings of the parameters. We also compare alternative instrument reduction strategies in a context where several endogenous variables enter the model. We find that the system GMM estimator is the best-performing in this framework and the high instrument count comes out not to be problematic. We also make the algebraic links between the parameters in the CAPB and in the PB models explicit, suggesting an effective strategy to estimate the discretionary fiscal response from the coefficients of the PB model. In the empirical application on a dataset for EMU Countries, we find that the evidence of a-cyclicality of discretionary policies is robust to all the specifications of the GMM estimator.
\end{abstract}

Keywords: Fiscal reaction functions, Monte Carlo simulations, dynamic panel data analysis, generalized method of moments, reduction of instruments count. JEL classification: C15, C33, E62, H60.

*Department of Economics, University of Bologna. E-mail: irene.mammi@unibo.it. This paper draws on chapter 3 of the author's PhD dissertation prepared at IMT Institute for Advanced Studies Lucca. The author thanks her supervisor Giorgio Calzolari and the board of examiners for their comments. The author is also especially indebted to Roberto Golinelli for his guidance and useful suggestions and for having provided the data used in the empirical application. 


\section{Introduction}

The empirical estimation of fiscal reaction functions has gained in popularity in the last few decades and it is now particularly diffused in the fiscal policy literature. The estimation of fiscal rules, enhanced by the seminal works of Bohn [1998] and Taylor [2000], has been mainly motivated by the question whether discretionary fiscal actions by the policymakers act pro-cyclically or countercyclically.

Finding an answer about the reaction of budgetary policies to the economic cycle has become particularly important within the European Monetary Union where the constraints imposed by the Maastricht Treaty and the Stability Growth Pact potentially affect the response of fiscal variables to the economic cycle and could weaken the autonomy of the national policymaker in determining discretionary fiscal actions.

In a very influential paper, Galì and Perotti [2003] find that discretionary policy in the EMU Countries has become more countercyclical over time: their work has given a further boost to a lively debate on the response of fiscal policies to the cycle that has led to many recent contributions which estimate fiscal reaction function in the $\mathrm{EU}$ or the OECD area. Among the most relevant works it is worth numbering in particular the contributions of Ballabriga and Martinez Mongay [2002], Balassone and Francese [2004], Forni and Momigliano [2004], Wyplosz [2006], Debrun and Kumar [2007], Golinelli and Momigliano [2006] and Beetsma and Giuliodori [2008]. What emerges from this stream of literature, however, is a strong lack of consensus on whether discretionary fiscal policy behaves pro-cyclically or counter-cyclically: the results are often conflicting, even in the case they focus on similar, or even same, samples of Countries and on a comparable time span. Golinelli and Momigliano [2009] provide an extensive survey of the empirical works on fiscal response functions and explain the huge heterogeneity in empirical findings in terms of differences in the specifications of the fiscal rule, in the estimation methodologies and in the samples covered.

In a context where the empirical findings have proved not to be robust to different estimation methodologies, we believe it can be very useful to investigate the sensitivity of the estimates of the fiscal rules' coefficients to alternative estimation techniques. Our main interest is therefore to make a comparison among alternative estimators that can be adopted for the estimation of fiscal response functions.

The dimension of the datasets exploited in the empirical fiscal policy literature is generally limited both in terms of the number of years available and in terms of Countries considered: it is therefore becoming common habit to consider the sample as a short panel and to adopt panel data techniques to estimate fiscal rules. The fiscal reaction function is by its nature a dynamic model which also includes unobservable time-invariant individual-specific effects, potentially

endogenous additional regressors and in which the series of interest are likely to 
be highly persistent. The choice of the methodology should therefore account for these relevant econometric issues and try to fix them properly. Unfortunately, in spite of these problems, it is still common to estimate the coefficients of fiscal reaction functions by pooled OLS or fixed-effect estimators ${ }^{1}$ that may not be appropriate in this context.

A common approach in the literature to address these econometric issues in this context is the use of a linear Generalized Method of Moments (GMM) estimator, the most common specification being the Arellano-Bond Difference GMM estimator [Arellano and Bond, 1991] for dynamic panel data models ${ }^{2}$. However, in the context of fiscal policy, where the series are know to be often very persistent, the Blundell-Bond System GMM estimator [Blundell and Bond, 1998] might be a more appropriate choice, for its allowing to overcome a potential weak instrument problem only requiring mild stationarity assumptions for the regressors. Up to now, fiscal reaction functions have been estimated by System GMM only in few empirical works ${ }^{3}$. Overall there is still very little guidance on which is the safest strategy to adopt for the estimation of fiscal response function.

The main purpose of the paper is to compare the performances of different dynamic panel data estimators in the estimation of fiscal rules and, in particular, to investigate the sensitiveness of the estimates to alternative specifications of the GMM estimator. We pay a particular attention to the issues of endogeneity intrinsic in this context and of the related high number of potentially exploitable moment conditions. In this respect, we address the problem of instrument proliferation by adopting specific techniques aimed at reducing the instrument count and we investigate the robustness of the estimates to these alternative specifications of the matrix of moment conditions.

The underlying relationships among the variables that enter fiscal reaction functions come from national accountancy rules: the data generating process is known relatively well and no additional behavioural assumption is needed. Thanks to that, also the sources of endogeneity are easily identified, so that proper instrumental variables can be exploited.

The present work contributes to the literature in fiscal policies in several ways.

First, we simulate the most popular fiscal rule estimated in the literature, namely the cyclically-adjusted primary balance $(C A P B)$ model $^{4}$, and we use Monte Carlo experiments in order to assess the performance of alternative estimators. To our knowledge, this is the first Monte Carlo experiment on fiscal

\footnotetext{
${ }^{1}$ Taylor [2000], Galì and Perotti [2003], Forni and Momigliano [2004], Wyplosz [2006], Debrun et al. [2008], among others, adopted these strategies.

${ }^{2}$ This approach has been followed, among the others, by Balassone and Francese [2004], Forni and Momigliano [2004], Debrum and Kumar [2007].

${ }^{3}$ Golinelli and Momigliano [2006, 2009] and Bernouth et al. [2008] are among the few ones.

${ }^{4}$ In a nutshell, the $C A P B$ model aims at explaining the discretionary fiscal policy (i.e. not due to automatic stabilizers) on the basis of an economic cycle indicator and of the initial conditions of both deficit and debt.
} 
rule models that aims at comparing various estimators and at giving practical indications on the safest methodologies to use in this framework. As far as we know, only Celasun and Kang [2006] have used Monte Carlo simulations for the estimation of fiscal reaction functions ${ }^{5}$.

Secondly, we estimate on simulated data also a different fiscal rule, the primary balance $(P B)$ model $^{6}$, where the dependent variable is the unadjusted primary balance, in order to check whether assessing the discretionary fiscal response to the cycle directly from the estimates of the $P B$ model is a safe strategy.

Third, we estimate the $C A P B$ model on real data for the EMU and we present the estimates of the model's coefficients obtained by means of a bunch of alternative estimators.

The remainder of the paper proceeds as follows. In section 2 we present in details the simulation model and we discuss the setting of the parameters in the fiscal rule. Section 3 introduces relevant econometric issues and reports the estimates of the simulated $C A P B$ model. In section 4 we illustrate the $P B$ model and its links with the $C A P B$ model, we estimate it on simulated data and we present a strategy to derive the discretionary adjustments to the cycle from the estimates of the $P B$ model parameters. Section 5 is devoted to the estimation of the $C A P B$ model on real data. Section 6 draws conclusive indications and sketches potential addresses for future research.

\section{The simulation model}

Our reference model here is the fiscal reaction function used in most empirical works in fiscal policy ${ }^{7}$ : the $\mathbf{C A P B}$ model, where the dependent variable, namely the change in the cyclically-adjusted primary balance $(\triangle C A P B)$, measures the discretionary fiscal actions that can be taken by policy makers. $\triangle C A P B$ is explained by the lagged values of the cyclically-adjusted primary balance $(C A P B)$ and the stock of public debt $(D E B T)$, that represent the state of public finances, and by the economic cycle as captured by the lagged

\footnotetext{
${ }^{5}$ More in detail, Celasun and Kang [2006] estimate a fiscal rule where the dependent variable is the primary balance, rather than the cyclically-adjusted primary balance, and where the lagged dependent variable is not included among the regressors. On the one hand, they aim at assessing the bias of the OLS and of the Least Squares Dummy Variable (LSDV) estimators for the coefficients of the fiscal rule with respect to the bias that rises in a standard $A R(1)$ model for the debt; on the other hand they aim to check the robustness of the estimates to different parameter settings in a static fiscal rule. We argue, in line with most of the literature, that the dynamics is an intrinsic feature of fiscal reactions function and that it can not be disregarded.

${ }^{6}$ Differently from the $C A P B$, the $P B$ model aims at explaining the overall fiscal policy, instead of only the discretionary policy.

${ }^{7}$ The $C A P B$ model is chosen, among the others, by Galì and Perotti [2003], Forni and Momigliano [2004], Wyplosz [2006] and Golinelli and Momigliano [2009].
} 
level of the output gap $(G A P)^{8}$.

The fiscal rule we estimate is the following:

$$
\triangle C A P B_{i t}=\phi_{c} C A P B_{i, t-1}+\phi_{d} D E B T_{i, t-1}+\phi_{g} G A P_{i, t-1}+\mu_{i}+\varepsilon_{i t}
$$

where the $\mu_{i} \sim \mathcal{N}\left(0, \sigma_{\mu}^{2}\right)$ are the time-invariant country-specific effects for the $C A P B$ and the $\varepsilon_{i t} \sim \mathcal{N}\left(0, \sigma_{\varepsilon}^{2}\right)$ are fiscal policy shocks. A positive coefficient $\phi_{g}$ implies that discretionary budgetary actions are counter-cyclical, while a negative coefficient indicates pro-cyclicality.

It follows from equation (1) that the $\mathrm{AR}$ process for the $C A P B$ is:

$$
C A P B_{i t}=\left(\phi_{c}+1\right) C A P B_{i, t-1}+\phi_{d} D E B T_{i, t-1}+\phi_{g} G A P_{i, t-1}+\mu_{i}+\varepsilon_{i t} .
$$

We thus need to simulate all the variables that appear in equation (2): in order to dispose of the series for the gap, the debt and the cyclically-adjusted primary balance, we also need to simulate additional variables from which the variables of interest are derived, according to accountancy rules. We generate the output gap as an autoregressive process that does not include other fiscal variables, but it is affected by fiscal policy shocks that also affect other variables; the cyclically-adjusted primary balance is generated according to the process in equation (2) and the remaining variables follow well-known public accountancy rules.

Our simulation model is as follows.

\subsection{Generating the variables of interest}

We initialize the $C A P B$ by generating its initial conditions so that they are stationary as follows:

$$
C A P B_{i 0}=\frac{\mu_{i}}{1-\phi_{g}}+u_{i 0}
$$

where the $u_{i 0} \sim \mathcal{N}(0,1)$ are the random deviations from the long-run mean of the cyclically-adjusted primary balance $\mu_{i} /\left(1-\phi_{g}\right)$.

Once we have the initial value for $C A P B$ and the initial values of the variables that appear in equation (2), we are able to generate $C A P B_{i 1}$ and so on, iteratively, period by period.

We generate stationary initial conditions also for the output gap as follows:

$$
G A P_{i 0}=\frac{\eta_{i}}{1-\alpha}+z_{i 0}
$$

\footnotetext{
${ }^{8}$ We take here the output gap lagged one period as common in the literature. It is worth noticing however that some authors include the simultaneous output gap instead of the lagged one. At the same way, some authors prefer the actual level of the $C A P B$ as dependent variable instead of its change. Golinelli and Momigliano [2009], in their extensive review of the literature on the empirical estimation of fiscal reaction functions, discuss the different specifications of the fiscal models, the links between them and how the interpretation of the estimation results changes according to the model chosen in the analyses.
} 
where the $\eta_{i} \sim \mathcal{N}\left(0, \sigma_{\eta}^{2}\right)$ are unobserved time-constant effects for the output gap and $z_{i 0} \sim \mathcal{N}(0,1)$ are the deviations from the long-run mean of the output gap.

We then generate the rest of the series as follows:

$$
G A P_{i t}=\alpha G A P_{i, t-1}+\eta_{i}+\xi \varepsilon_{i, t-1}+v_{i t}
$$

where the $\varepsilon_{i t}$ are again the fiscal policy shocks and the $v_{i t} \sim \mathcal{N}\left(0, \sigma_{v}^{2}\right)$ are idiosyncratic shocks.

The nominal growth rate $n_{t}$ is created as follows:

$$
n_{t} \equiv\left[e^{\ln \left(1+\dot{p}_{i t}\right)+\ln \left(1+p g_{i t}\right)+\Delta \ln \left(1+G A P_{i t}\right)}\right]-1
$$

where $\dot{p}_{i t}$ is the inflation rate and $p g_{i t}$ is the potential growth. The inflation rate follows the process $\dot{p}_{i t}=\dot{p}_{i, t-1}+\zeta G A P_{i, t-1}$, with $\zeta=0.05$, while the potential growth is assumed to be centered around the $2 \%$ according to the process $p g=2+\gamma_{i t}$ with $\gamma_{i t} \sim \mathcal{N}(0,1)$ being a random shock.

The primary balance variable $P B_{i t}$ is obtained as:

$$
P B_{i t} \equiv \frac{C A P B_{i t}}{1+G A P_{i t}}+\omega \frac{G A P_{i t}}{1+G A P_{i t}}
$$

with $\omega$ being the elasticity of the overall budget that represents the effect of the automatic stabilizers.

Interest payments are generated as follows:

$$
I N T_{i t} \equiv R_{i t} \frac{D E B T_{i, t-1}}{1+n_{i t}}
$$

where $R_{i t}$ is the average cost of debt and is assumed to move together with the nominal growth according to the process $R_{i t}=n_{i t}+\iota_{i t}$ with $\iota_{i t} \sim \mathcal{N}(0,1)$ being a random shock.

We can then obtain the overall fiscal balance as:

$$
B_{i t} \equiv P B_{i t}-I N T_{i t} .
$$

Finally, we can generate the public debt as follows:

$$
D E B T_{i t}=\frac{D E B T_{i, t-1}}{1+n_{i t}}-B_{i t} .
$$

\subsection{Baseline setting for the parameters}

We simulate data for a fixed number of periods $T$ and for a fixed number of economies $N$. Our reference framework is that of advanced economies such as the Countries in the European Union or the most developed OECD Countries ${ }^{9}$.

\footnotetext{
${ }^{9}$ Our choice is motivated by the fact that the European Union, and in particular the Euro area, is a privileged context for the estimation of fiscal reaction functions in the fiscal policy literature and it will also be our sample in the empirical analysis in the prosecution of the paper.
} 
The number of Countries is set to $N=15$ : this in line with most of the empirical literature on fiscal reaction functions in which the sample size is generally not far from 15. The number of time periods in the estimating sample is set to $T=15$. Such time span allows for a dynamic panel data analysis, without rising serious concerns about poolability of the series. In order to have 15 periods available, we generate data for a time span of 50 periods and we allow for different presamples, i.e. 1, 15 and 35 years. Independently of the length of the pre-sample, the generated 50-years samples are always the same: what changes is simply the point in history we capture in the analysis. Through calibration, we set the means and the standard deviations of the generated variables as well as the parameters in the model to values that make the simulated scenario as realistic as possible so that the profiles of the simulated series are close to the observed ones for the EMU Countries, or are plausible realizations of actual data for advanced economies.

In Table (1) we summarize the baseline setting of the parameters that are used in the core of our simulation exercise. Deviations of several parameters from the baseline values have also been considered to check for the robustness of the results ${ }^{10}$.

Table 1: Baseline setting of the parameters in the simulation model

\begin{tabular}{|l|l|}
\hline \hline$N$ & 15 \\
\hline$T$ & 15 \\
\hline$\sigma_{\mu}$ & 0.15 \\
\hline$\sigma_{\eta}$ & 0.30 \\
\hline$\sigma_{\varepsilon}$ & 0.30 \\
\hline$\sigma_{v}$ & 1 \\
\hline$\phi_{c}+1$ & 0.8 or 0.1 \\
\hline$\phi_{g}$ & $0.10,-0.10$ or 0 \\
\hline$\phi_{d}$ & 0.15 \\
\hline$\alpha$ & 0.8 or 0.1 \\
\hline$\xi$ & 0.2 \\
\hline$\omega$ & 0.5 \\
\hline \hline
\end{tabular}

We generate the values of the variables as percentages of the GDP so that, e.g., a value for $D E B T_{i t}$ equal to 50 indicates that the stock of public debt of Country $i$ in period $t$ is the $50 \%$ of the GDP; therefore, according to the same scaling, a standard deviation equal to 1 has to be seen as a standard deviation of $1 \%$. With regards to our baseline setting, we can notice from Table (1) that the standard deviations for the unobserved time-invariant effects of the output gap

\footnotetext{
${ }^{10}$ The results are not presented here but are available upon request. In particular, we have run the experiments with larger variances for the time-invariant Country-specific effects of $C A P B$ and $G A P$ and the fiscal policy shocks, that is $\sigma_{\mu}=\sigma_{\eta}=\sigma_{\varepsilon}=1$. The profile of the series, with this modified setting, become explosive and the degree of heterogeneity introduced in the data is unrealistic.
} 
and the cyclically-adjusted primary balance are relatively low. This is justified by the fact that, in our period by period simulation process, the Country-specific effects are cumulated year after year through the autoregressive processes of the two variables. Standard deviations between 0.15 and 0.30 are high enough to introduce in the model a realistic degree of heterogeneity that matches closely the standard deviation (around 0.40) of the unobserved individual effects estimated on actual data. Higher variances for the $C A P B$ and $G A P$ effects would imply an induced non-stationarity in the series and an explosive profile for the debt $^{11}$. We keep also the variance of the fiscal policy shocks relatively low, as the shocks are generated separately and independently for each period and each Country. In order not to complicate the model further, we do not assume here that common shocks are present among the Countries neither we introduce any transmission mechanism of the shocks ${ }^{12}$. The simultaneous fiscal policy shock appears in equation (2) for $C A P B$, while the lagged fiscal shock shows up in the equation for $G A P$, scaled by a coefficient $\xi=0.2$, thus making the output gap predetermined but not strictly exogenous.

In our baseline simulation, we set the autoregressive coefficients for $G A P$ and $C A P B$ both to 0.8 , as the actual series are very persistent ${ }^{13}$. As a pure robustness check for our results, we also consider an opposite extreme scenario in which the dynamics of the gap is very fast $(\alpha=0.1)$ and the inertia of fiscal policies is lower, i.e. $\phi_{c}+1=0.1$. We acknowledge that such an alternative setting is certainly far from being realistic but it can be very useful for robustness checks.

We fix $\omega$, the effect of the automatic stabilizers, to 0.5 as constant over time and across Countries: the actual value of this elasticity is around 0.5 on average in the Euro area and in the advanced OECD economies ${ }^{14}$ so this is the most natural choice in our simulation, as commonly used in the literature.

A crucial issue is the setting of the parameters of the variables in the fiscal rules, as they represent the reaction of discretionary fiscal actions, as measured by $\triangle C A P B$, to the economic cycle and to the initial fiscal conditions. When the policies are sticky, i.e. $\phi_{c}+1=0.8$, the implied $\phi_{c}$ in equation (1) is -0.2 ; when policies are instead completely flexible, i.e. $\phi_{c}+1=0.1$, we have $\phi_{c}=-0.9$.

We expect $\phi_{g}$ and $\phi_{d}$ not to be very high in absolute values, as the effect of discretionary actions is by its nature limited when compared to the effect

\footnotetext{
${ }^{11}$ In this latter case, unrealistic values for the unobserved individual-specific effects variances generate a trend in the series.

${ }^{12}$ In fact, it would then be unrealistic to assume that very severe fiscal shocks could affect only one Country without affecting the other economies.

${ }^{13}$ The dynamics of the economic cycle, measured by $\alpha$, is generally very slow; the inertia of the fiscal policies, measured by the autoregressive coefficient of the $C A P B$, i.e. $\phi_{c}+1$, is commonly very strong.

${ }^{14}$ The average $\omega$ for the Members of the EU-15 is generally between 0.45 and 0.50 and it is on average around 0.45 for the New Members of the EU. See IMF Fiscal Monitor [2011] and OECD Economic Outlook [2011] for further details.
} 
of automatic stabilizers. The chosen values for $\phi_{g}$ and $\phi_{d}$ are respectively 0.1 and $0.005^{15}$. The choice about the value of $\phi_{g}$ is also a relevant issue. Setting a coefficient $\phi_{g}=0.10$ implies the assumption that discretionary fiscal decisions are strongly counter-cyclical, while they could also be pro-cyclical or even a-cyclical. As the main aim of our simulation exercise is to compare the performances of alternative estimators rather than to check whether the fiscal policies are cyclically symmetric or not, what matters here is the absolute value of the output gap coefficient, rather than its sign. As a robustness check, we also run simulations with $\phi_{g}=-0.10$. An interesting case is also to assume that fiscal policy are a-cyclical and set $\phi_{g}=0$, in order to investigate whether the alternative estimators are able to detect such a-cyclicality.

\section{Monte Carlo experiments for the CAPB model}

\subsection{Relevant econometric issues}

The model of interest, as specified in equation (1), is a dynamic model for panel data in which time-invariant Country-specific effects are allowed and the variables are generally highly persistent.

The output gap in a fiscal rule is likely not to exogenous; accounting for that, in the simulation model, the output gap is generated as predetermined but not strictly exogenous, due to the presence in its generating process of the lagged fiscal shocks that also appears in the fiscal rule in their realisation contemporaneous to $\triangle C A P B_{i t}$. Such endogeneity of the output gap has therefore to be tackled in the estimation procedure. A similar reasoning also holds true for the public debt, which can be hardly assumed as exogenous to shocks in the $C A P B$ : in our simulated model, the debt is by construction endogenous and needs therefore to be instrumented. A different kind of endogeneity is due to the dynamic specification of the fiscal rule: the lagged dependent variable is correlated with the Country-specific effects in the model, so that it is not strictly exogenous, giving rise to the well-known dynamic panel bias. Both the within transformation and the first difference transformation, the latter being also a pillar of GMM estimation, induce a correlation between the transformed lagged dependent variable and the transformed error terms and thus imply endogeneity problems in the model. An instrumental variable approach is therefore needed in this framework. In the GMM-estimation of the model, we will therefore instrument all the regressors. We need to be aware that the recourse to instruments

\footnotetext{
${ }^{15}$ It is also worth noticing that, despite the fact that the adjustment to the output gap and to initial fiscal conditions seems small at a first sight, these parameters imply a long run adjustment relationship as follows:

$$
C A P B^{*}=\frac{0.1}{0.2} G A P^{*}+\frac{0.005}{0.2} D E B T^{*}=0.5 G A P^{*}+0.025 D E B T^{*}
$$

that is far from being economically irrelevant.
} 
for all the regressors can easily lead to a problem of instrument proliferation that could bias the GMM estimates ${ }^{16}$ and weaken the tests for overidentifying restrictions. It is therefore opportune to try to detect this problem and adopt proper solutions to face it.

Another relevant issue is that the most realistic simulated scenario, with $\phi_{g}=0.8$ and $\phi_{c}+1=0.8$, implies a high degree of persistence of the series of interest. In this context, the weak instrument issue could easily arise in the difference-GMM estimation of the model as the instruments in levels are only weakly correlated to the lagged first-differenced endogenous variables ${ }^{17}$.

\subsection{Estimation results on simulated data}

We now present the outcomes of the Monte Carlo exercise, which allow to analyze the performance of alternative estimators for the coefficients of the fiscal rule in equation (1).

For each setting of the parameters in the simulation model, we run 1000 iterations on samples with 225 observations and report the mean of each estimated coefficient over all the iterations (mean), the standard deviation of the estimated coefficient $(s d)$, the power of the t-test for each coefficient $(s i g)^{18}$ and the average Hansen-test $p$-value (hansenp).

Equation (1) is estimated with the following panel data estimators (the abbreviations used in the tables are within brackets): Ordinary least squares $(O L S)$; Fixed-effects estimator $(F E)$; Anderson-Hsiao estimator $(A H)$ using only instruments in levels; Arellano-Bond difference-GMM estimator exploiting the full set of moment conditions $(A B a)$; Blundell-Bond system-GMM estimator exploiting the full set of moment conditions $(B B a)^{19}$; Arellano-Bond differenceGMM estimator with a limited set of instruments $(A B l)$ which only uses the $t-2$ and $t-3$ lags of the endogenous regressors as instruments; Blundell-Bond system-GMM estimator with a limited set of instruments $(B B l)$ which only uses the $t-2$ and $t-3$ lags of the endogenous regressors as instruments for the equation in first differences; Arellano-Bond difference-GMM estimator with a collapsed set of instuments $(A B C)$ in which the full instrument set is collapsed following Roodman [2009a]; Blundell-Bond system-GMM estimator with a collapsed set of instruments $(B B C)$; Arellano-Bond difference-GMM estimator with a limited collapsed set of instruments $(A B l c)$ where the limited set

\footnotetext{
${ }^{16}$ See Ziliak [1997], Roodman [2009a] and Bowsher [2002] for further references.

${ }^{17}$ See Blundell and Bond [1998] for the discussion of this well-known issue.

${ }^{18}$ For each coefficient, we test the null hypotesis $H_{0}: \beta=0$ against the alternative hypothesis $H_{a}: \beta \neq 0$ at a $5 \%$ significance level. The power of the test gives us the probability of rejecting $H_{0}$ when it is false. Only in the case of simulated data for which $\phi_{g}=0$, sig should be interpreted as the size of the test, as it gives the probability of rejecting $H_{0}$ when it is true.

${ }^{19}$ In this specification, all the potentially available lags from $t-2$ backward are used as instruments in levels for the model in first differences and the lagged first-difference of the endogenous regressors is used as instrument for the equation in levels.
} 
of instruments is also collapsed; Blundell-Bond system-GMM estimator with a limited collapsed set of instruments $(B B l c)$; Arellano-Bond difference-GMM estimator with an instrument set reduced by means of the Principal Component Analysis $(\mathrm{PCA})^{20}$ according to the average criterion $(A B p c a a)^{21}$; Arellano-Bond difference-GMM estimator with an instrument set reduced by means of the PCA according to the variability criterion $(A B p c a v)^{22}$; Blundell-Bond system-GMM estimator with an instrument set reduced by means of the PCA according to the average criterion (BВpсаa); Blundell-Bond system-GMM estimator with an instrument set reduced by means of the PCA according to the variability criterion (BBpcav). The dependent variable is always the change in the cyclically-adjusted primary balance, namely $\triangle C A P B_{i t}$. Since all the regressors are considered as not strictly exogenous, they are all instrumented. All the estimates are made robust to the potential heteroskedasticity of the disturbances. With respect to the GMM estimator, only one-step estimates of the parameters are reported ${ }^{23}$. The true parameters for the lagged $D E B T$ and GAP are respectively $\phi_{d}$ and $\phi_{g}$ whose values are set as in Table (1); with respect to the lagged value of the dependent variable instead, its true parameter will be $\phi_{c}=-0.2$ when we set $\phi_{c}+1=0.8$ and $\phi_{c}=-0.9$ when $\phi_{c}+1=0.1$. The core of our simulations is run on a 15-year pre-sample so that the length of the pre-sample equals the number of periods considered in the analysis.

The estimation results for the $C A P B$ model are presented through Table (2) to Table (7).

For the sake of brevity, in the comments to the estimates we will refer to DIFF and SYS GMM to indicate, respectively, the system and the difference GMM. UNTR, COL and LIM will indicate respectively that the estimators exploit the untransformed, collapsed or limited ${ }^{24}$ set of instruments. PCA stands for the set of instruments reduced by means of $\mathrm{PCA}^{25}$. When two reduction techniques are combined, two abbreviations are also combined (e.g. LIMCOL).

We adopt a 15 -year pre-sample in our experiments. As a preliminary robustness check, however, Table (2) and Table (3) present the estimation results when we set either a 1-year pre-sample or a 35 -year pre-sample. The setting of the parameter is the baseline one, with $\phi_{c}=0.8$ and $\phi_{g}=0.8$. In general, what

\footnotetext{
${ }^{20}$ For details on the the application of PCA on the GMM-style instrument matrix see Bontempi and Mammi [2012].

${ }^{21}$ We keep in the analysis only the principal components whose eigenvalues are above the average of the eigenvalues.

${ }^{22}$ We keep a number of principal components such that the explained variance is the $70 \%$ of the total variance in the original data.

${ }^{23}$ Though more efficient in large samples and well performing in simulated samples, the two-step estimator suffers a poor finite sample performance and it is rarely used in empirical analyses. We also estimated all the coefficient by two-step GMM and found larger biases and variances due to a small sample. Two-steps results are available upon request.

${ }^{24}$ We sometimes specify 1 or 2 to indicate a lag depth of 1 or 2 .

${ }^{25}$ The additional letters $\mathrm{A}$ and $\mathrm{V}$ will indicate, respectively, that the principal components are retained according to the average and the variability criteria.
} 
emerges in this first scenario is that the behavior of the alternative estimators is sufficiently in line with what is generally expected in a dynamic panel data framework, in the case of both a short and a very long pre-sample: the GMM estimators perform better than the OLS estimator, as the latter does not take into account the presence of Country-specific time-invariant effects, while the FE estimator, that accounts for this heterogeneity, gives results closer to the GMM estimates, to the Arellano-Bond in particular. With respect to the estimates of the parameter of interest $\phi_{g}$, we find that different specifications of the DIFF estimator tend in general to perform slightly better in terms of mean than the alternative specifications of the SYS estimator when the pre-sample is shorter, though the variance is smaller for the SYS GMM estimators: such behaviour is reversed with a longer pre-sample, with the SYS GMM estimator still having the lowest variance. The SYS estimator, however, seems to have the best performance overall: this could be due to the fact that, once the initial conditions satisfy the Blundell-Bond assumptions, it is less sensitive to the accumulation of shocks and individual heterogeneity over time that is intrinsic in our simulation process. An evident feature is the under-rejection of $H_{0}$ for $\phi_{g}$ by the DIFF estimator: this problem becomes more serious as the number of instruments decreases and useful information is dropped from the instrument matrix. In DIFF GMM frameworks, the instruments are weak if the series are persistent and a reduction in their count comes out to be problematic. With respect to $\phi_{c}$, the behaviour of the alternative estimators is the standard one for dynamic panel data models: the OLS estimator overestimates the coefficient, the DIFF GMM is systematically biased downwards, while the SYS estimator is the best performing, being less biased, in particular when the pre-sample is short. It is worth stressing the good performance of alternative SYS PCA estimators, especially for the estimate of $\phi_{g}$, and, on the contrary, the very poor behaviour of the DIFF PCA estimators. In a dynamic model with very persistent series, the reduction of the DIFF GMM instrument matrix through PCA further worsens the weak instrument problem already present. When the instruments are relevant, as for the SYS GMM estimator, the use of PCA to reduce the instrument count does not worsen the estimates. As far as $\phi_{d}$ is concerned, in both the scenarios the most evident feature is the very misleading under-rejection of $H_{0}$ : only in a minority of cases the coefficient is detected as significant, as it actually is. Overall, the adjustment of fiscal policy to the initial public debt is either overestimated or underestimated and it is in general estimated far from the true value of 0.005 .

Interestingly, the Hansen test $p$-values are far from $1^{26}$ only for LIM COL estimates, suggesting a severe and generalized problem of instrument proliferation and invalid over-identifying restrictions. However, such lower Hansen test $p$-value does not come together with a significant improvement of estimators

\footnotetext{
${ }^{26}$ The implausible $p$-value of 1 is interpreted as a symptom of instrument proliferation problems, as argued in Roodman [2009a].
} 
performance with respect to the UNTR estimates; rather, it comes along with an under-rejection of the null hypotheses of non significant coefficients.

\subsubsection{Sticky policies and very persistent gap: $\phi_{c}+1=0.8$ and $\alpha=0.8$}

In Table (4) we report the results of our baseline scenario with a pre-sample of 15 years. We have very persistent economic cycle and very persistent fiscal policies.

With respect to $\phi_{g}$, the OLS estimator not surprisingly gives the most biased estimates because it neglects the presence of Country-specific effects, that are by construction very relevant in this framework. The DIFF and SYS GMM UNTR estimators give similar estimates, though the SYS estimator has systematically a lower variance. When the instrument count is reduced, the performance of the DIFF estimators suffers a severe worsening, while the SYS estimators do not change significantly their behaviour. The bad performance of the DIFF estimators with transformed instrument sets is generally accompanied by a severe under-rejection of $H_{0}$ of $\phi_{g}=0$. In parallel, the SYS PCA estimator gives the least biased estimates overall, while the DIFF PCA is the estimator that suffers the most. Applying the PCA on the DIFF GMM set of weak instruments further worsens the weak instrument problem due to very feeble covariances between the instruments. By adding the instruments in first differences, we overcome this weakness issue and we have valid instruments that can be safely reduced in their count: in fact, SYS PCA estimates are better than SYS UNTR estimates. It is remarkable the fact that the Hansen test systematically gives an implausible $p$-value of 1 or very close to 1 , except for LIM COL estimates: the more reliable $p$-value, though not really "safe" yet ${ }^{27}$, does not come along with a sensible improvement in the estimates, that are worse than the LIM estimates. The collapsing of the instrument matrix is not effective in this context and gives less robust estimates. In other terms, the number of instruments unavoidably becomes very large in this context, but this does not seem to affect significantly the estimates: the UNTR GMM or, at most, the LIM estimator ${ }^{28}$ perform well and a further reduction in the instrument count by collapsing does not seem required or can be misleading at worst. Considering the estimates for $\phi_{c}$, we find a high degree of heterogeneity in the estimates and features that are typical in GMM estimation of dynamic panel data models: the OLS estimator, that ignores the individual heterogeneity, seriously underestimates the dynamics of the $C A P B$, by severely overestimating the coefficient; the DIFF estimators tend to systematically estimate a too low coefficient and have very high standard deviations. Things improve a lot when the coefficients are esti-

\footnotetext{
${ }^{27}$ Roodman [2009a] suggests on worrying whenever a $p$-value above 0.25 is obtain, as higher values are symptoms of a weakening of the tests on over-identifying restrictions due to instrument proliferation.

${ }^{28}$ The lag depth truncation is very common in the empirical literature whenever we have a large number of endogenous regressors.
} 
mated by SYS GMM, as this estimator is both less biased and more efficient. The SYS LIM and the SYS PCA estimates are the closest to the true value of $\phi_{c}$. The collapsing of the instrument matrix comes along with more biased estimates and, when associated also with the limitation of the lags, with a marked under-rejection of the null hypothesis for $\phi_{c}$. The reduction of the instrument set through PCA in DIFF GMM appears to be very dangerous because of a worsening of weak instrument problems. The tendencies already underlined for the output gap coefficient are exacerbated when the coefficient of the lagged dependent variable is considered. In a context characterized by stickiness of the fiscal policies and a high persistence of the output gap, it appears reasonable to estimate the adjustment effects by SYS UNTR or by SYS LIM GMM estimators: the instrument proliferation problem does not appear to be a true issue in fiscal rule estimation and in our simulations there is not evidence of a concrete risk of overfitting of the endogenous variables, despite the controversial results of the Hansen test. With respect to $\phi_{d}$, it is confirmed that there is a general tendency to under-reject $H_{0}: \phi_{d}=0$ and that the estimates are often not sufficiently close to the true value, except for the SYS estimates, which are also those with the lowest variance.

\subsubsection{Less persistent policies and gap: $\phi_{c}+1=0.1$ and $\alpha=0.1$}

In Table (5) we consider a less realistic scenario where $\alpha=0.1$, so that the economic cycle is very fast, and $\phi_{c}+1=0.1$ is such that the fiscal policies present almost no inertia at all. The aim of the simulation with this particular setting of the parameters is to check whether the previous findings are robust to different degrees of persistence of the series. The true values of $\phi_{g}$ and $\phi_{c}$ are respectively 0.10 and -0.90 .

As far as $\phi_{g}$ is concerned, the behaviour of the alternative estimators is in line with that found in the baseline scenario, with SYS UNTR and the SYS PCA being the best performing estimators and the ones with the lowest variance. Overall, a slight difference is that the estimates for the output gap generally tend to be closer to the true value of $\phi_{g}$ : since here the output gap is set as not persistent, the weak instrument problem is mitigated and the DIFF GMM estimates are less affected than in the previous scenario. Also the tendency to under-reject the null hypothesis on $\phi_{g}$ is alleviated and the COLL estimates, even in the case of the DIFF estimators, are closer to the true value. In a less persistent scenario, the estimates are less sensitive to the specifications of the GMM estimators and they are not worsened by the adoption of instrument reduction techniques. It is remarkable however the bad performance of the DIFF PCA estimator in this case: if the series are close to being white noise, the lagged levels of the endogenous regressors have very weak covariances between them and are not suitable to undergo the PCA. In general, if the series were not persistent, every choice about the specification of the GMM estimators would be relatively safe and would give reliable estimates. When the series are very 
persistent, the issue of instrument weakness seem to dominate that of instrument proliferation. If the instruments are weak, a reduction in their number through the collapsing of the instrument matrix can bring the estimates far from the true values of the coefficients. On the other hand, when the instruments are stronger, the reduction of the instrument count has a very little impact on the estimates. The estimates of $\phi_{c}$ follows the same regularities as above: the OLS estimator overestimates $\phi_{c}$ and has a very high variance in the experiment; the DIFF estimators overestimate the effect of the lagged $C A P B$ by giving a too large coefficient. When the $C A P B$ is less persistent, $\phi_{c}$ is always detected as statistically significant. In this last scenario, the estimation of $\phi_{d}$ comes out to be the most problematic, as the parameter of the debt is very often stated not significant, especially when the SYS GMM estimator is used; the DIFF GMM tends to find the debt statistically significant many more times, but this is due to the fact that it generally overestimates the adjustment effects of the fiscal policies to the initial stock of debt. From the analysis of the results in Table (2) through Table (5), the sign of $\phi_{g}$ is always correctly estimated by the GMM estimator; on the other hand, the estimates of $\phi_{g}$ point to an a-cyclicality of discretionary fiscal policies too often.

\subsubsection{Alternative cyclicality of the policy stance: $\phi_{g}=-0.10$ and $\phi_{g}=0$}

As a further robustness check, in Tables (6) and (7) we consider again the scenario characterized by the high persistence of both the gap and the $C A P B$, but we set respectively $\phi_{g}=0$ and $\phi_{g}=-0.10$, so that discretionary fiscal policies are assumed to be a-cyclical in the first case and pro-cyclical in the second one.

With respect to $\phi_{c}$ and $\phi_{d}$ we find that in both cases there are not relevant differences with respect to the results presented above. The same is also true for the Hansen test $p$-value that is still in line with the values found before. What we are most interested in here is whether and how there are changes in the estimates of $\phi_{g}$.

In the scenario in which $\phi_{g}=0$, we can check for the size of alternative estimators through the frequency of rejection of the null hypothesis of a non significant coefficient for the output gap. We have here an over-rejection of $H_{0}$, as the size of the test is above the $5 \%$. In particular, the estimates tend too often to a pro-cyclicality or to a counter-cyclicality of the fiscal policies when they are actually a-cyclical. The SYS COL estimator tends to exacerbate this problem. However, this tendency is not as marked as the one, detected above, to find a-cyclicality when the policies are actually not neutral to the economic cycle.

When $\phi_{g}=-0.10$, the estimates are generally in line with that in the case in which $\phi_{g}=0.10$, though they are slightly less biased and the tendency to under-reject $H_{0}$ is less severe. In particular, the OLS estimator is much closer 
to the true parameter and the DIFF estimates are more similar to the SYS ones. It is confirmed that the SYS PCA estimator performs impressively well in a context where fiscal policies are very sticky. The adoption of instrument reduction techniques does not necessarily improve the estimate, though it lowers the Hansen-test $p$-value.

Overall, the main conclusion we draw from these Monte Carlo experiments is that even when the best performing estimator, the SYS GMM in all its variants, is used, it is frequent to detect a misleading reaction of discretionary fiscal policies to the economic cycle.

\section{Estimation of the PB model}

Up to now, we have focused on the CAPB model with the aim of estimating the cyclical reaction of discretionary fiscal policies.

Another approach commonly adopted in the literature is the estimation of alternative fiscal response functions that focus on the reactions of the overall budget. Among these models, the PB model aims at estimating the overall reaction of fiscal policies to the economic cycle, rather than the discretionary responses only as in the case of the $C A P B$ model. In the $P B$ model, the dependent variable, which represents the decision of the policymaker, is the change in the unadjusted overall primary balance, while the explanatory variables are given by the lagged primary balance, the lagged debt and the lagged output gap. The $P B$ model reads therefore as follows:

$$
\triangle P B_{i t}=\phi_{p}^{P B} P B_{i, t-1}+\phi_{d}^{P B} D E B T_{i, t-1}+\phi_{g}^{P B} G A P_{i, t-1}+u_{i t}
$$

where $u_{i t}$ is an error terms that collects unobserved individual heterogeneity, fiscal policy shocks and idiosyncratic errors.

The primary balance is, by definition:

$$
P B_{i t} \equiv C A P B_{i t}+\omega_{i t} G A P_{i t},
$$

that is the sum of the cyclically-adjusted primary balance, that captures the structural or discretionary budget, and a cyclical component given by the product of the output gap and the effect of automatic stabilizers. It is therefore straightforward too see that the change in the primary balance measures the full fiscal action.

The main parameter of interest is in this case $\phi_{g}^{P B}$, as it gives indications on whether fiscal policies are pro-cyclical, in case of a negative sign, or countercyclical, in case of positive coefficient.

As the PB model allows to estimates only gross effects, it is common in the fiscal policy literature to estimate the reaction of discretionary policies to the cycle by subtracting the coefficient $\omega^{29}$, that is the average of $\omega_{i t}$, from

${ }^{29} \omega$ has generally a value around 0.5 . 
the estimated coefficient of the output gap in the PB model $\left(\phi_{g}^{P B}\right)^{30}$. The cyclicality of discretionary policies is thus estimated as:

$$
\phi_{g}^{P B(d i s c r)} \approx \phi_{g}^{P B}-\omega .
$$

We estimate the $P B$ model in equation (12) on the simulated data. We run 1000 iterations, exploit the same estimators used for the $C A P B$ and consider all the regressors as endogenous so that they are all instrumented. The baseline settings used for the $C A P B$ model are maintained. In Tables (8) and (9) we report the estimates for the scenario in which both the gap and the cyclicallyadjusted primary balance are very persistent and in which the two processes are almost not persistent at all. In this framework, the true value of the coefficient for the output gap in the $C A P B$ is set to 0.10 . In the tables we also report the estimates of $\phi_{g}^{P B(d i s c r)}$ that are implied by equation (14); we use $\omega=0.5$ in all our experiments. For convenience's sake, in the last rows of Tables (8) and (9) we report the estimates of $\phi_{g}^{C A P B}$ obtained in exactly the same experiment setting ${ }^{31}$. Both in the more persistent and in the less persistent scenarios, the estimates of $\phi_{g}^{P B}$ are not very different from those obtained for $\phi_{g}^{C A P B}$ : when we use the alternative specifications of the SYS GMM, we get estimates for $\phi_{g}^{P B}$ that are in the range $0.06-0.09$, not very far from those in the range $0.095-0.11$ obtained by estimating the CAPB model. In other words, the estimated $\phi_{g}^{P B}$ is far from being higher than the estimated $\phi_{g}^{C A P B}$ of about 0.5. This gets evident when we consider the estimates of $\phi_{g}^{P B(d i s c r)}$ : if subtracting $\omega$ from the estimated $\phi_{g}^{P B}$ were a correct strategy in order to estimate the discretionary reaction in the $\mathrm{PB}$ model, we would expect the estimated $\phi_{g}^{P B(d i s c r)}$ to have an average close to $\phi_{g}^{C A P B}=0.10$. This is not the case here as we systematically get a very large negative estimate of $\phi_{g}^{P B(d i s c r)}$ : this would point to a very strong pro-cyclicality of discretionary fiscal policies in a context in which the policies have been set as counter-cyclical. These evidences are generally confirmed also in the less persistent framework.

We have argued that the most realistic profile is the one where the gap and the deficit are strongly persistent: we find here that, in this context, it can be very misleading to estimate the discretionary effects according to equation (14) because of the risk of drawing wrong conclusions about the cyclicality of the policies, no really matters which estimator is used. In order to estimate the reaction of discretionary policies to the economic cycle, the best strategy is to estimate $\phi_{g}^{C A P B}$.

\footnotetext{
${ }^{30}$ See, among the others, Bouthevillain et al. [2001], Balassone and Franzese [2004] and Socol and Socol [2009].

${ }^{31}$ These estimates are from Tables (4) and (5).
} 


\subsection{Algebraic links between the parameters of the PB model and the CAPB model}

We aim here at discovering the reasons why the estimates of $\phi_{g}^{C A P B}$ and of $\phi_{g}^{P B}$ do not differ as much as we would expect.

We recall here that the $C A P B$ model is:

$$
\triangle C A P B_{i t}=\phi_{c}^{C A P B} C A P B_{i, t-1}+\phi_{d}^{C A P B} D E B T_{i, t-1}+\phi_{g}^{C A P B} G A P_{i, t-1}+\mu_{i}+\varepsilon_{i t}
$$

and that the $P B$ model is:

$$
\begin{aligned}
\Delta P B_{i t}= & \phi_{p}^{P B} P B_{i, t-1}+\phi_{d}^{P B} D E B T_{i, t-1} \\
& +\phi_{g}^{P B} G A P_{i, t-1}+u_{i t} .
\end{aligned}
$$

By exploiting the equivalence $P B_{i t} \equiv C A P B_{i t}+\omega G A P_{i t}$, we can derive the exact relationships between the coefficients in the two models.

By substituting the expression for $P B_{i t}$ into the $P B$ model we have, through some algebraic manipulations:

$$
\begin{aligned}
\Delta C A P B_{i t}= & \phi_{p b}^{P B} C A P B_{i, t-1}+\phi_{d}^{P B} D E B T_{i, t-1}+ \\
& \phi_{p b}^{P B} \omega G A P_{i, t-1}+\phi_{g}^{P B} G A P_{i, t-1}- \\
& \omega G A P_{i t}+\omega G A P_{i, t-1}+v_{i t}
\end{aligned}
$$

It is straightforward that the coefficient of $C A P B_{i, t-1}$ in the $C A P B$ model is the same as the coefficient of $P B_{i, t-1}$ in the $P B$ model and that the coefficient of the lagged debt is the same in the two models. We thus have:

$$
\begin{aligned}
& \phi_{p b}^{P B}=\phi_{c}^{C A P B} \\
& \phi_{d}^{P B}=\phi_{d}^{C A P B}
\end{aligned}
$$

In order to derive an analogous link for the gap coefficient, we need to substitute $\alpha G A P_{i, t-1}+\eta_{i}+\xi \varepsilon_{i, t-1}+v_{i t}$ as in equation (5) for $G A P_{i t}$. We get:

$$
\begin{aligned}
\Delta C A P B_{i t}= & \phi_{p b}^{P B} C A P B_{i, t-1}+\phi_{d}^{P B} D E B T_{i, t-1}+ \\
& +\left(\omega \phi_{p b}^{P B}+\phi_{g}^{P B}+\omega-\alpha \omega\right) G A P_{i, t-1} \\
& -\omega\left(\eta_{i}+\xi \varepsilon_{i, t-1}+v_{i t}\right)+u_{i t} .
\end{aligned}
$$

The relationship between the gap parameters in the two models is therefore the following:

$$
\phi_{g}^{C A P B}=\omega \phi_{p b}^{P B}+\phi_{g}^{P B}+\omega(1-\alpha)
$$

Note also that both $\phi_{p b}^{P B}$ and $\alpha$ play a role in this relationship and are crucial in order to determine how close the estimates in the two models are. 
We have just seen that $\phi_{p b}^{P B}=\phi_{c}^{C A P B}$ so that, if for example we set $\omega=0.5$ and $\phi_{c}^{C A P B}+1=0.8$, we will get $\phi_{p b}^{P B}=\phi_{c}^{C A P B}=-0.2$ from which $\omega \phi_{p b}^{P B}=$ -0.1 . When the autoregressive coefficient of the output gap is set to $\alpha=0.8$, we have that $\omega \phi_{p b}^{P B}=\omega(1-\alpha)=0.1$ : in this case, the output gap parameters in the two models are the same, i.e. $\phi_{g}^{C A P B}=\phi_{g}^{P B}$ as the first and the last terms cancel out. Whenever the dynamics of the primary balance and of the output gap are similar, the two terms will tend to annul each other and estimates of the coefficients for the gap in the two models very close each other are expected. For the relationship $\phi_{g}^{P B(\text { discr })} \approx \phi_{g}^{P B}-\omega$ to be reasonable, the dynamics of the two processes are required to be approximately the same in magnitude but with opposite signs ${ }^{32}$. It is evident that such a scenario is not realistic.

After having made the link between the coefficients explicit, we can go back to the estimation results for the PB model in Tables (8) and (9). In the tables the implied $\phi_{g}^{C A P B}$ as deriving from equation (20) are reported ${ }^{33}$. Imposing the same dynamics for the primary balance and for the output gap, we obtain estimates of the gap coefficient in the $P B$ model close to those in the $C A P B$ model, though they are not as close as we would expect according to equation (20). The estimates of the gap are more heterogeneous than the respective ones in the CAPB model and present a much higher variance. When compared to the estimates for $\phi_{c}^{C A P B}$ in Tables (3) and (4), the estimates for $\phi_{p b}^{P B}$ are much more volatile than those in the $C A P B$ and are generally far from the value of -0.2 we would expect according to equation (17). In the most persistent scenario, the behaviour of the estimators reflects the one we have underlined in the $C A P B$ estimation: the OLS estimator largely underestimates the effect by overestimating the coefficient, the DIFF GMM estimators gives too large coefficients, while the SYS GMM estimators are the best and the most efficient. The SYS UNTR and the SYS PCA give estimates close to -0.2 and with the smallest variance overall. In the case in which $\alpha=0.1$ and $\phi_{c}^{C A P B}+1=0.1$ we have less heterogeneity in the estimates and an overall better performance of the DIFF estimators over the SYS ones: in a less persistent framework, the weak instrument problem is mitigated and parsimony in the number of instruments is beneficial.

Overall, the estimates of the coefficients in the $P B$ model are more biased and display a much larger variance than those for the $C A P B$ model: this reflects in an incorrect implied $\phi_{g}^{C A P B}$ coefficient as derived from the $P B$ estimates. As both the estimates of $\phi_{p b}^{P B}$ and $\phi_{g}^{P B}$ enter equation (17), if the are biased and imprecise, the estimate of $\phi_{g}^{C A P B}$ will also be likely to be biased. If the estimates

\footnotetext{
${ }^{32}$ The relationship would hold, for example, if the primary balance were a static process and the output gap were a unit root process or if $\phi_{g}^{C A P B}+1=x$ and $\alpha=-x$.

${ }^{33} \mathrm{It}$ is worth saying that here we generate the $P B$ series according to equation (7), that is we scale both $C A P B$ and $G A P$ by the term $1+G A P$, so that the relationship in equation (13) does not perfectly hold: the denominator, however, is very close to 1 , as the gap moves within the range $-5 / 5 \%$, so we should not expect very significant deviations in the estimates.
} 
of $\phi_{p b}^{P B}$ and $\phi_{g}^{P B}$ were better, we would expect that the relationship $\phi_{g}^{P B}=$ $\phi_{g}^{C A P B}$ would approximately hold, as the two terms $\omega \phi_{p b}^{P B}$ and $\omega(1-\alpha)$ would tend to cancel out. Here, instead, the dynamics of the primary balance and the response to the gap are estimated very imprecisely and the implied $\phi_{g}^{C A P B}$ is therefore a very weak, and often misleading, estimate of the discretionary response to the gap. This larger bias and imprecision of the estimates in the $P B$ model could be explained by the fact that the $P B$ model estimates gross unadjusted effects and introduces much more noise in the variables, with respect to the $C A P B$ model that filters out the cycle effects from the primary balance. We can have an intuition of that by looking at the term $-\omega\left(\eta_{i}+\xi \varepsilon_{i, t-1}+v_{i t}\right)$ in equation (19): this noise is part of the error term in the $C A P B$ model, while it is part of the $P B$ variable in the $P B$ model. Overall measures of the deficit are therefore more likely to be endogenous to the shocks in the model than already adjusted measures.

For the sake of completeness, we report in Tables (10) and (11) the results for the alternative mixed scenarios $\alpha=0.8 ; \phi_{c}+1=0$. and $\alpha=0.1 ; \phi_{c}+1=0.8$. In this cases we give the $C A P B$ and the $G A P$ different dynamics and we expect to find estimates of $\phi_{g}^{P B}$ far from 0.10 as the terms $\omega \phi_{p b}^{P B}$ and $\omega(1-\alpha)$ do not cancel out anymore. This is exactly what happens, as the estimates of the gap coefficient are centered respectively around 0.30 and -0.20 : in both cases the estimate of $\phi_{g}^{(P B) d i s c r}$ is very misleading as it implies a very strong procyclicality of the discretionary policies, especially in the second scenario, when they are instead set as counter-cyclical. The estimate of the implied $\phi_{c}^{C A P B}$ is less biased, but only when the SYS GMM estimator is used. Neither in this case, however, the strategy of using equation (17) in order to estimate the discretionary response to the gap is completely safe, due to the large heterogeneity in the estimates of the coefficients $\phi_{g}^{P B}$ and $\phi_{p b}^{P B}$.

In conclusion, if we want to assess how discretionary policies react to the cycle, the safest strategy is the estimation of $\phi_{g}^{C A P B}$ directly in the $C A P B$

model by SYS GMM. Moreover, it is not reasonable to estimates $\phi_{g}^{(P B) d i s c r}$ by subtracting $\omega$ from the estimated $\phi_{g}^{P B}$ : in case it is only possible to estimate the $P B$ model, it is advisable to estimate the discretionary response by applying the strategy suggested in this section.

\section{Estimation of the CAPB model on real data}

We estimate the $C A P B$ model on the dataset used in Golinelli and Momigliano [2009] and we adopt the alternative specifications of the GMM estimator already employed in our simulations. The sample includes data for 11 Countries of the European Monetary Union over the period 1994-2008. Data for the cyclically adjusted primary balance and the output gap are taken from the OECD Economic Outlook [n. 83, June 2008]; data for the stock of public debt derive from 
the European Commission AMECO database [June 2008] $]^{34}$. We choose the specification of the $C A P B$ model as in Table (4) of Golinelli and Momigliano [2009] where, in addition to the lagged dependent variable, debt and gap, we include two additional explanatory variables that are by now standard in empirical analysis of fiscal rules: an election variable $(\text { Elect })^{35}$, that is a dummy variable equal to 1 in years in which regular elections take place (0 otherwise), and a Maastricht variable (Maas), capturing the role of EU rules, that is different from 0 when the deficit is above the 3 per cent target threshold ${ }^{36}$.

The model we estimate on real data is therefore:

$$
\begin{aligned}
\triangle C A P B_{i t}= & \phi_{c} C A P B_{i, t-1}+\phi_{d} D E B T_{i, t-1}+\phi_{g} G A P_{i, t-1} \\
& +\beta \text { Maas }_{i, t-1}+\delta \text { Elect }_{i t}+\mu_{i}+\tau_{t}+\varepsilon_{i t}
\end{aligned}
$$

where $\mu_{i}$ are unobserved individual-specific effects, $\tau_{t}$ are time effects and $\varepsilon_{i t}$ is the idiosyncratic term.

We estimate the model in equation (21) by GMM and by OLS $^{37}$. All the estimates include time dummies. In line with Golinelli and Momigliano [2009] and with the previous Monte Carlo experiments, we take the lagged dependent variable, the output gap and the Maastricht variable as endogenous: these three variables are therefore instrumented by their own lags in levels in the equation in first-differences and by their lagged first difference in the equation in levels. With respect to the debt, we adopt two alternative strategies: first, following Golinelli and Momigliano [2006], we consider the debt as an exogenous regressor $^{38}$; second, we consider also the debt as endogenous and we instrument it with GMM-style instruments ${ }^{39}$. We keep the same abbreviations as in Section 3.2 for the alternative estimators in the tables: however, when we lag-truncate

\footnotetext{
${ }^{34}$ Details on the definitions of the variables and on the sources of data are in the Appendix of Golinelli and Momigliano [2009].

${ }^{35}$ Data are from the International Institute for Democracy and Electoral Assistance (IDEA).

${ }^{36}$ The Appendix of Golinelli and Momigliano [2009] provides technical details on the construction of the Maastricht variable.

${ }^{37}$ Our main interest is in GMM estimation of fiscal response functions with alternative specification of the GMM estimator. We therefore omit here the FE estimates, as they are supplanted by the Arellano-Bond estimator in empirical analysis, and Anderson-Hsiao estimates, as they have proved to be greatly biased also in a controlled experiment. These additional results are available upon request.

${ }^{38}$ In this perspective, the potential endogeneity of the debt in an economic sense does not translate into an endogeneity in a pure statistical sense, as it happens instead in the case of the lagged dependent variable; therefore the debt does not necessarily need to be instrumented. This strategy is in line with the common habit of using GMM-style instruments only for the lagged dependent variable when other potential endogenous regressors are not endogenous in a statistical sense. See, e.g., Arellano and Bond [1991].

${ }^{39}$ In our simulations, the debt was endogenous by construction, as generated sequentially in such a way that it incorporated fiscal policy shocks, economic cycle shocks and individual effects, and it necessarily needed to be instrumented. In a real world, we are inclined to believe the debt to be truly correlated with fiscal policy shocks and to the economic cycle, and therefore necessarily correlated with the error term. We thus prefer to consider it endogenous.
} 
the instrument matrix, we consider both the case in which only one lag is kept ( $l 1$ and $l c 1$ stand for the limited and collapsed matrix $)^{40}$ and the case, as above, in which we keep two lags of each endogenous variable ( $l 2$ and $l c 2$ for the limited and collapsed matrix). All the estimates are made robust to the potential heteroskedasticity of the errors; GMM estimates are always one-step. In each table, we report the estimate of the coefficient, the standard error and the $t$-statistic.

We want first to replicate the results in column (4) of Table (4) in Golinelli and Momigliano [2009]; we then estimate the model by all the alternative estimators. In the original paper, the estimates are not made robust to heteroskedasticity; they are obtained by one-step SYS GMM and use only the first available lag for the endogenous regressors ${ }^{41}$; as hinted above, the authors consider the debt exogenous.

We use the command xtabond2 for GMM estimation of dynamic panel data in $\mathrm{Stata}^{42}$ in the latest available version for Stata 12. The authors obtained their estimates in Stata 9 with the release of the xtabond2 command available at the time the paper was published.

In Table (12), in the first column we replicate the original estimate not robust to heteroskedasticity; in the second column we make it robust using the version of xtabond2 exploited by the authors; in the third and the fourth columns we replicate the estimates of the first and the second ones by using the latest version of xtabond2.

In Table (12), we do not find any difference in the point estimates of the coefficients, while we have discrepancies in the estimated standard errors only when the estimates are not made robust to heteroskedasticity of the errors. Inference can be affected by whether or not we account for potential heteroskedaticity: in the case of the lagged dependent variable, e.g, we find a coefficient significant at a $10 \%$ significance level when estimates are not robust, not significant when they are robust.

In Table (13) we report the results for the model in equation (21) when the debt is assumed to be exogenous. A noticeable heterogeneity in the estimates of the coefficient $\phi_{g}$ is found: there is not any agreement on the estimated sign of the coefficient and the response to the gap is never found to be statistically significant. The estimates are particularly imprecise and unreliable, since they are characterized by a very large standard error, in particular when any technique is adopted in order to reduce the number of instruments. In line with the findings in our Monte Carlo simulations, the SYS GMM estimators tend to give more counter-cyclical coefficients than the DIFF GMM estimators and estimates with a smaller variance. This is particularly evident for the LIM COL estimates. When we look at the $t$-statistic, all the estimators point to the acyclicality of the discretionary fiscal policies. With respect to the coefficient of

\footnotetext{
${ }^{40}$ This choice is made in line with Golinelli and Momigliano [2009] who use only the first valid lag for the regressors.

${ }^{41}$ The original estimate is the $B B l 1$ in our notation.

${ }^{42}$ Details about the syntax are in Roodman [2009b].
} 
the lagged dependent variable, in line with the Monte Carlo experiment, the DIFF estimators systematically tend to give larger coefficients than the SYS estimators and larger standard errors. There is a strong tendency of the estimates to become less negative or even positive when we adopt LIM, COL or LIMCOL estimators. The smaller the transformed instrument set, the less negative the coefficient. This gets along with a propensity to go towards a non significance of the coefficient as more as we reduce the number of instruments: for the UNTR estimator we find an estimate of $\phi_{c}$ around -0.2 and the coefficient comes out to be strongly statistically significant; for the LIM2 GMM the estimates remain very close to the untransformed ones and strongly significant, both in the case of the SYS and DIFF GMM estimation; LIM1 GMM gives smaller estimated coefficients that also become not significant. The SYS COLL estimator returns not significant estimates. Things worsen when the LIMCOL estimator is used: for LIM1COL we get estimates of the coefficient with a positive $\operatorname{sign}^{43}$, though not significant. Mindful of the results in the Monte Carlo experiment, we are inclined to believe that the "true" value of $\phi_{g}$ is in a neighborhood of $-0.15 /-0.2$, close to the value estimated by the untransformed system-GMM estimator. As we know that the $C A P B$ is by its nature a dynamic and very persistent process, we expect a significant $\phi_{c}$ coefficient, and we are suspicious whenever the coefficient is detected as not significant.

A sensible reduction in the instrument count in this framework comes along with a too severe loss of information that results in an impossibility to obtain reliable estimates of the fiscal response function. The instrument reduction techniques proposed by Roodman [2009a] reveal themselves as potentially dangerous in the current context where the lack of information in the data is a crucial issue. On the other hand, it is worth noticing that, when we adopt a purely statistical technique (such as PCA) to reduce the number of instruments, we have a smaller reduction in the instrument count but we safeguard the significance of the coefficients and we get estimates in line with those obtained on the untransformed instrument set. The Hansen test gives systematically a $p$-value of 1 even when the instrument count is drastically reduced: in the light of the previous simulations we can argue that we should not worry too much about this value, as an excessive number of instruments is likely to be less dangerous than the loss of information deriving from a reduction in the instrument count. With respect to the debt coefficient, it is detected as significant only when estimated by DIFF GMM; this comes along with a larger absolute value of the coefficient when compared to the SYS GMM estimator.

The two additional regressors appear to have a relevant explanatory power as their coefficients are very large and are generally found to be strongly significant: in particular, the Maastricht variable is found to have a systematically significant

\footnotetext{
${ }^{43}$ It is worth reminding here that an estimate of $\phi_{c}$ with a positive sign would imply an autoregressive coefficient for the $C A P B$, given by $\phi_{c}+1$, that is above 1: we would deal with the unrealistic scenario of an explosive primary balance series.
} 
effect that is estimated around $-0.5 /-0.7$ in the best GMM specifications; the regular elections appear to play a relevant role, suggesting a systematic fiscal loosening in the election years ${ }^{44}$.

This evidence supports the idea that these additional variables have to appear in a fiscal response function. Additional variables generally not included in the standard $C A P B$ model, such as institutional factors or budgetary rules, could play a relevant role in explaining the discretionary response of fiscal policies and their omission could potentially affect the inference on the coefficients of the variables included.

We could argue that, in the EMU context, the discretionary action of the policy maker is driven more by the provisions of the Stability Growth Pact rather than by the output gap and the actual stock of debt.

In Table (14) we report the estimate for the $C A P B$ model in the case where also the debt is treated as an endogenous regressor. The outcome does not change much with respect to the previous case. The main parameter of interest, $\phi_{g}$, is again constantly not significant in all the cases except for $A B l 1 c^{45}$. With respect to $\phi_{c}$, the estimates mirror those in Table (13), with a considerable tendency to be statistically significant and around -0.20 when the SYS GMM estimator is used, and to become implausibly positive and not significant when the dimension of the instrument matrix is reduced to the minimum. Again, we are inclined to believe the true parameter is not far from -0.20 . With respect to $\phi_{d}$, we find a significant coefficient as estimated by DIFF GMM and also when the instrument set is reduced through PCA or contemporaneously limited and collapsed. In contrast with what happens for the other endogenous regressors, whose significance is affected by a reduction in the instrument count, the coefficient of debt gets more significant as we reduce the number of instruments. Overall, there is not a clear indication on the role of the initial state of finances, as captured by the debt, in determining discretionary fiscal actions: the estimates are not robust to the alternative choices about the instrument matrix. Even in this second case, the two explanatory variables are found to be very important in explaining the discretionary fiscal action and this evidence is robust to the estimator used.

\section{Conclusions}

This work compares alternative dynamic panel data estimators for the estimation of fiscal response functions. To this end, we run extensive Monte Carlo simulations where we allowed various parameters in the fiscal rule to change.

\footnotetext{
${ }^{44}$ The coefficient looses in significance only when the instrument count is drastically reduced.

${ }^{45}$ The statistical significance in this case comes along with an implausibly high estimated coefficient. The most likely explanation is again the bias due to a too drastic reduction in the number of instrument together with the well-known tendency of the DIFF estimator to overestimate the coefficients.
} 
First, we estimated the $C A P B$ model by different estimators, always accounting for the dynamic structure of the model, for the endogeneity of the regressors, and for the risk of instrument proliferation in GMM estimation. We found significant heterogeneity in the estimates in all the scenarios and we showed that the System GMM estimator is the safest in this context as it gives the least biased estimates and the lowest variance. We also warned about the risk of getting very biased and misleading estimates when strategies to drastically reduce the number of instruments were adopted. In case of multiple endogenous regressors, we could lower significantly the $p$-value of the Hansen test only by paying the risk of obtaining unreliable estimates. We found that the safest instrument reduction strategy is the purely data-driven one, namely the application of the PCA on the instrument set. From the Monte Carlo experiment, we drew the recommendation to be cautious in the interpretation of the estimated coefficients in a fiscal rule: the coefficients were often detected as not significant when they actually were; on the other hand, there was also a tendency to label a coefficient as significant when it actually was not.

Second, we estimated the $P B$ model on simulated data and we cast new light on the risks of assessing the discretionary response of fiscal actions to the cycle by simply subtracting the effect of automatic stabilizers from the estimated coefficient of the output gap in the $P B$ model. In fact, when the dynamics of the gap and of the $C A P B$ are close each other and the discretionary policies are set to be counter-cyclical, this strategy points systematically to a pro-cyclicality of the policies. We suggested an alternative way to estimate the discretionary response starting from the coefficients of the PB model and we found that it is generally safer than the commonly adopted one. However, we concluded that the best strategy in order to assess the response of discretionary policies to the cycle remains the direct estimation of the CAPB model.

Third, we estimated the $C A P B$ model on the dataset of Golinelli and Momigliano [2009] for the EMU Countries. We confirmed that the estimates obtained by SYS GMM estimation are the most reliable; a too drastic reduction in the instrument count through a huge limitation of the lag depth and the collapsing of the instrument set seemed harmful for the estimates as it led to an implausible non-significance of the coefficient of the lagged dependent variable. We found that the detected a-cyclicality of discretionary fiscal policies in this sample is robust to all the specifications of the estimators. Across all the estimators, we systematically found a relevant importance of the SGP rules and of the regular elections in explaining discretionary fiscal actions and we argued that we should care more about potentially relevant institutional factors that can not be omitted a priori. 


\section{References}

[1] Anderson, T.W., Hsiao, C. (1981) "Estimation of dynamic models with error components", Journal of the American Statistical Association, vol. 76 , pp. 598-606.

[2] Anderson, T.W., Hsiao, C. (1982) "Formulation and estimation of dynamic models using panel data", Journal of Econometrics, vol. 18, pp. 47-82.

[3] Arellano, M. and S.R. Bond (1991) "Some tests of specification for panel data: Monte Carlo evidence and an application to employment equations", Review of Economic Studies, vol. 58, pp. 277-297.

[4] Balassone, F. and F. Francese (2004) "Cyclical asymmetries in fiscal policy, debt accumulation and the Treaty of Maastricht", Bank of Italy, Temi di Discussione n. 531.

[5] Balassone, F., Francese, F. and S. Zotteri (2008) "Cyclical asymmetries in fiscal variables", Bank of Italy, Temi di Discussione n.671.

[6] Ballabriga, F. and C. Martinez-Mongay(2002) "Has EMU shifted policy", European Commission,DG for Economic and Financial Affairs, Economic Paper n.166.

[7] Beetsma, R. and M. Giuliodori (2008) "Fiscal adjustment to cyclical developments in the OECD: an empirical analysis based on real-time data", CEPR, Discussion Paper n. 6692.

[8] Bernoth, K., Hughes Hallett, A. and J. Lewis (2008) "Did fiscal policy makers know what they were doing? Reassessing fiscal policy with real time data", CEPR, Discussion Paper n. 6758.

[9] Blundell, R.W. and S.R. Bond (1998a) "Initial conditions and moment restrictions in dynamic panel data models", Journal of Econometrics, vol. 87 , pp. 115-143.

[10] Bohn, H. [1998] "The behavior of U.S. public debt and deficits", Quarterly Journal of Economics, vol. 113, pp. 949-963.

[11] Bontempi, M.E. and I. Mammi [2012] "A strategy to reduce the count of moment conditions in panel data GMM", Working Paper Department of Economics, University of Bologna, DSE WP n. 843.

[12] Bouthevillain, C., Cour-Thimann, P., van den Dool, G., Hernandez de Cos, P., Langenus, G., Mohr, M., Momigliano, S., and M. Tujula (2001) "Cyclically adjusted budget balances: an alternative approach", European Central Bank, Working Paper n. 77. 
[13] Bowsher, C. G. (2002) "On testing overidentifying restrictions in dynamic panel data models", Economics Letters, vol. 77, pp. 211-220.

[14] Calderon, C.A., Chong, A. and N.V. Loyaza (2002) "Determinants of current account deficits in developing countries", Contributions to Macroeconomics, vol. 2.

[15] Celasun, O., and J. S. Kang (2006) "On the properties of various estimators for fiscal reaction functions", IMF, Working Paper n. 182.

[16] Debrun, X. and M. Kumar (2007) "The discipline-enhancing role of fiscal institutions: theory and empirical evidence", $I M F$, Working Paper n. 171.

[17] Debrun, X., Moulin, L., Turrini, A., Ayuso-i-Casals, J., and M. Kumar (2008) "National fiscal rules", Economic Policy, April 2008, pp. 297-362.

[18] Galì, J. and R. Perotti (2003) "Fiscal policy and monetary integration in Europe", Economic Policy, vol. 18, pp. 533-572.

[19] Golinelli, R. and S. Momigliano (2006) "Real-time determinants of the fiscal policies in the Euro area", Journal of Policy Modeling, vol. 28, pp. 943-964.

[20] Golinelli, R. and S. Momigliano (2008) "The cyclical response of fiscal policies in the Euro area: why do results of empirical research differ so strongly?", Bank of Italy, Temi di Discussione n. 654.

[21] Golinelli, R. and S. Momigliano (2009) "The cyclical reaction of fiscal policies in the Euro area: the role of modeling choices and data vintages", Fiscal Studies, vol. 30, n. 1.

[22] IMF (2011) "Fiscal monitor", International Monetary Fund, April 2011.

[23] Mehrhoff, J., (2009) "A solution to the problem of too many instruments in dynamic panel data GMM", Discussion paper n. 1/2009, Deutsche Bundesbank.

[24] OECD (2011) "Economic Outlook", OECD, n. 89, June 2011.

[25] Roodman, D. (2009a) "A Note on the theme of too many instruments", Oxford Bulletin of Economics and Statistics, vol. 71, pp. 135-158.

[26] Roodman, D. (2009b) "How to Do xtabond2: An Introduction to "Difference" and "System" GMM in Stata", Stata Journal, Vol. 9, pp. 86-136.

[27] Socol, C. and A. G. Socol (2009) "The analysis of fiscal policy management in Romania: lessons for emerging countries", African Journal of Business Management, vol. 3, pp. 240-247. 
[28] Taylor, J. B. (2000) "Reassessing discretionary fiscal policy", Journal of Economic Perspectives, vol. 14, n. 3, pp. 21-36.

[29] Wyplosz (2006) "European Monetary Union: the dark sides of a major success", Economic Policy, vol. 21, pp. 208-261.

[30] Ziliak (2006) "Efficient estimation with panel data when instruments are predetermined: an empirical comparison of moment-condition estimators", Journal of Business and Economic Statistics, vol. 15, pp. 419-431. 


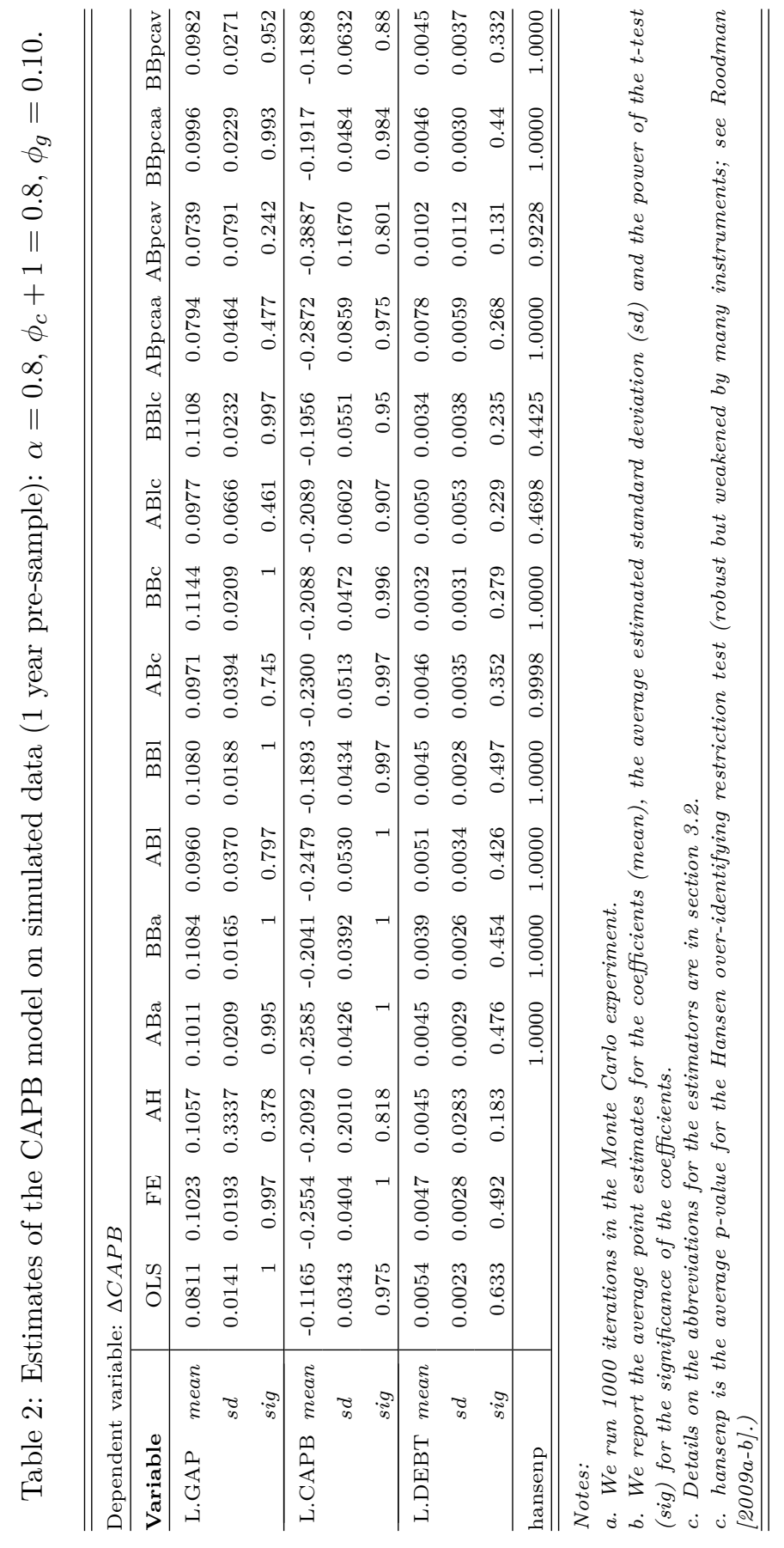




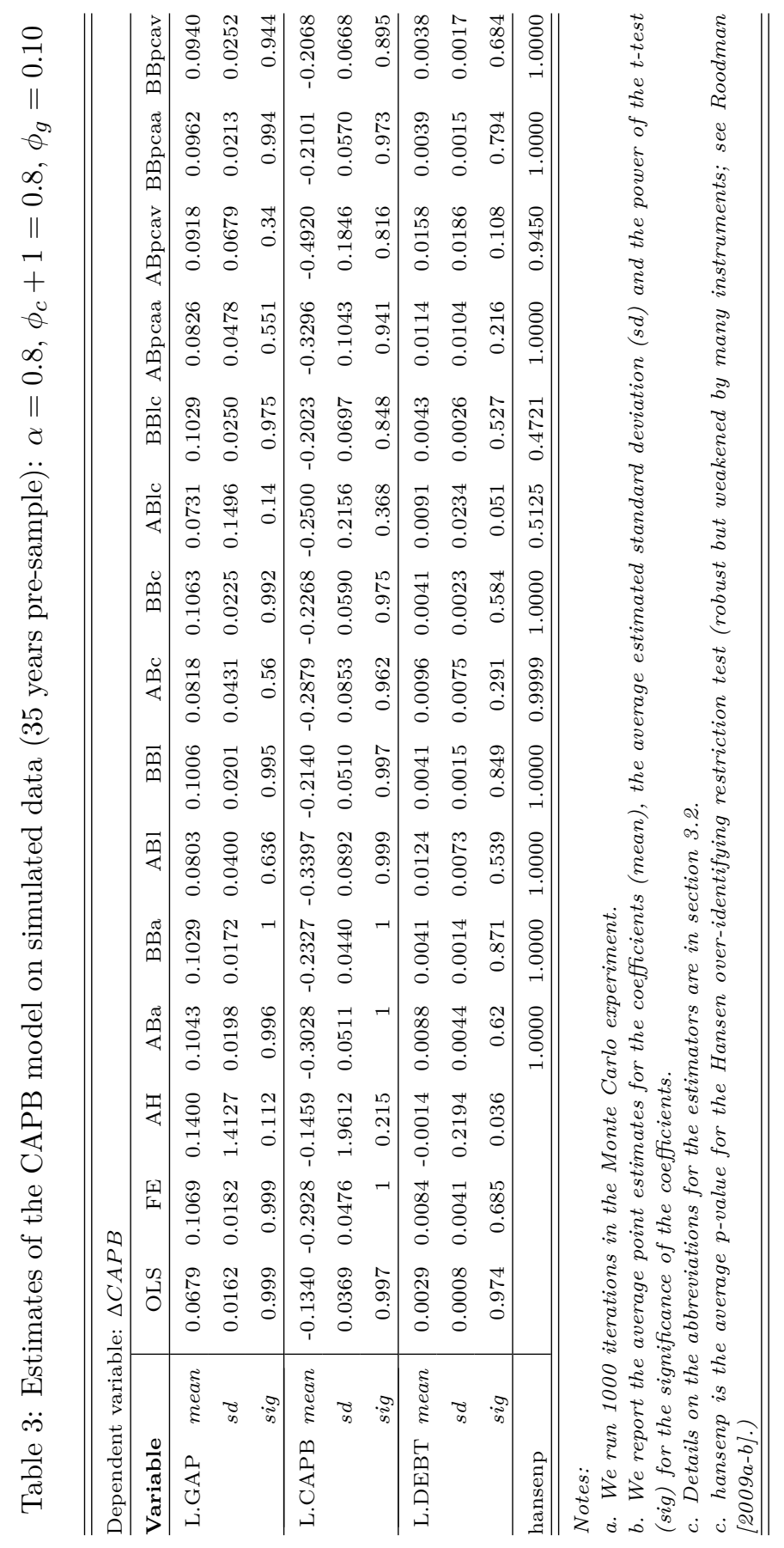




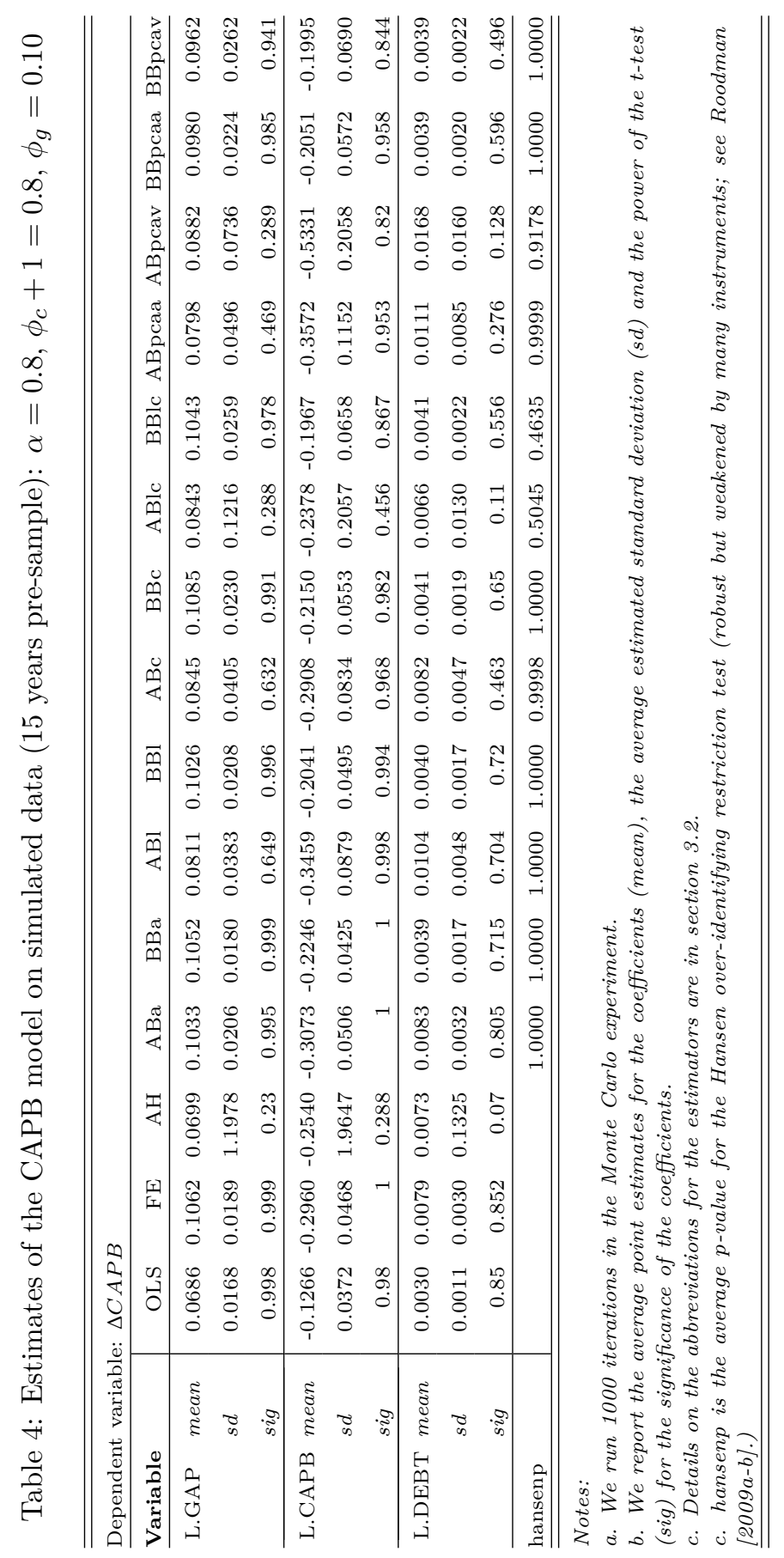




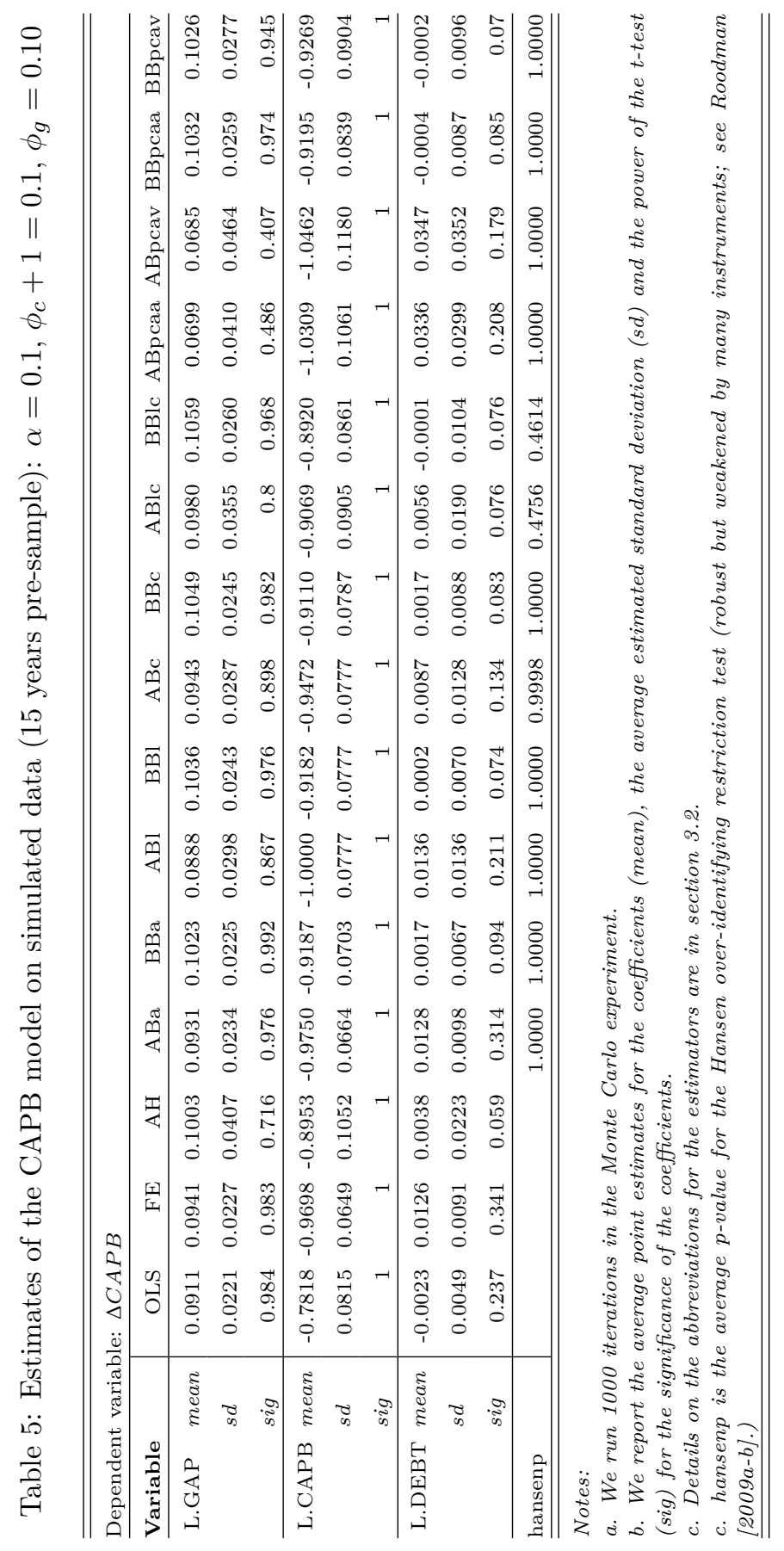




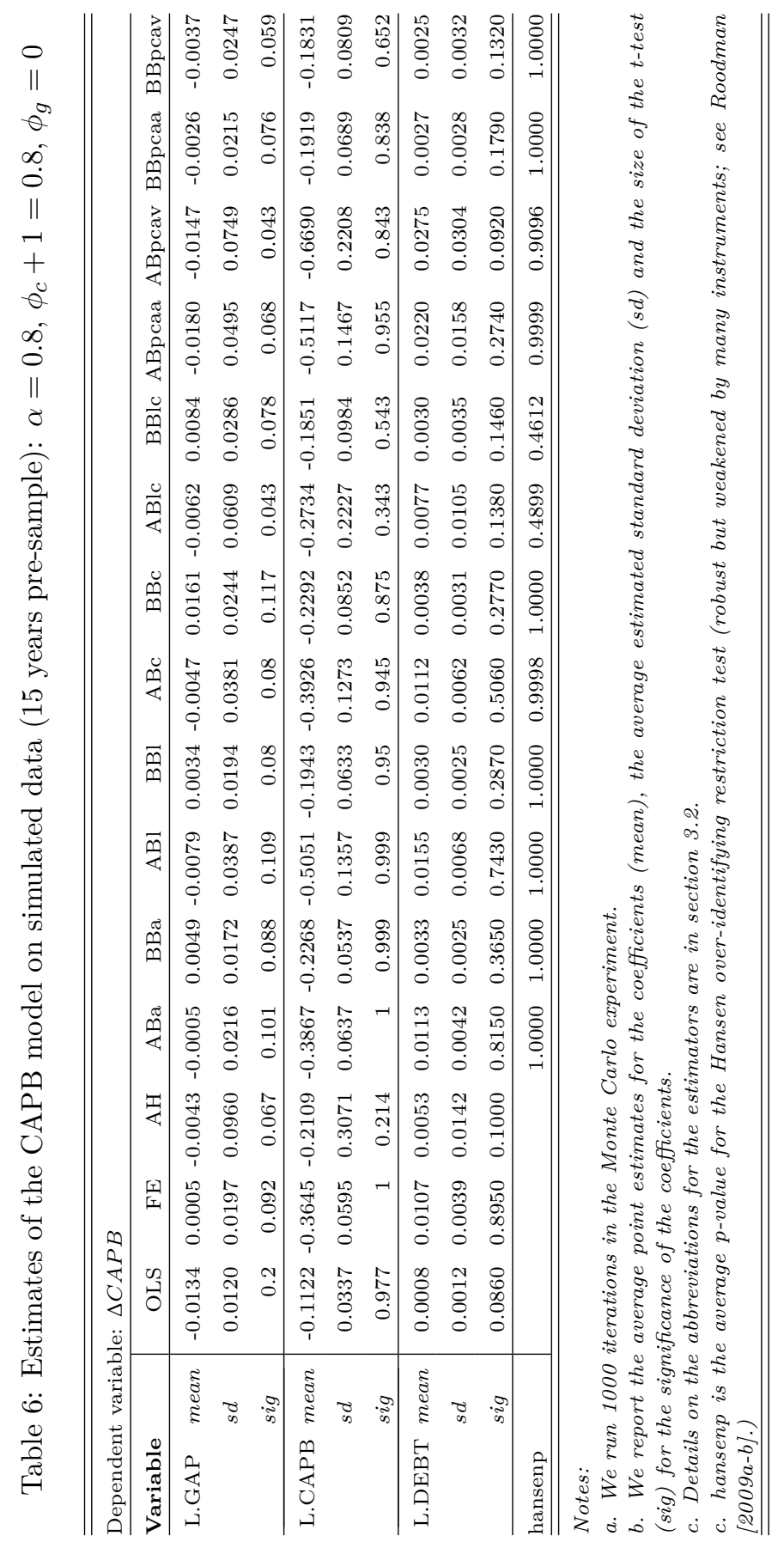




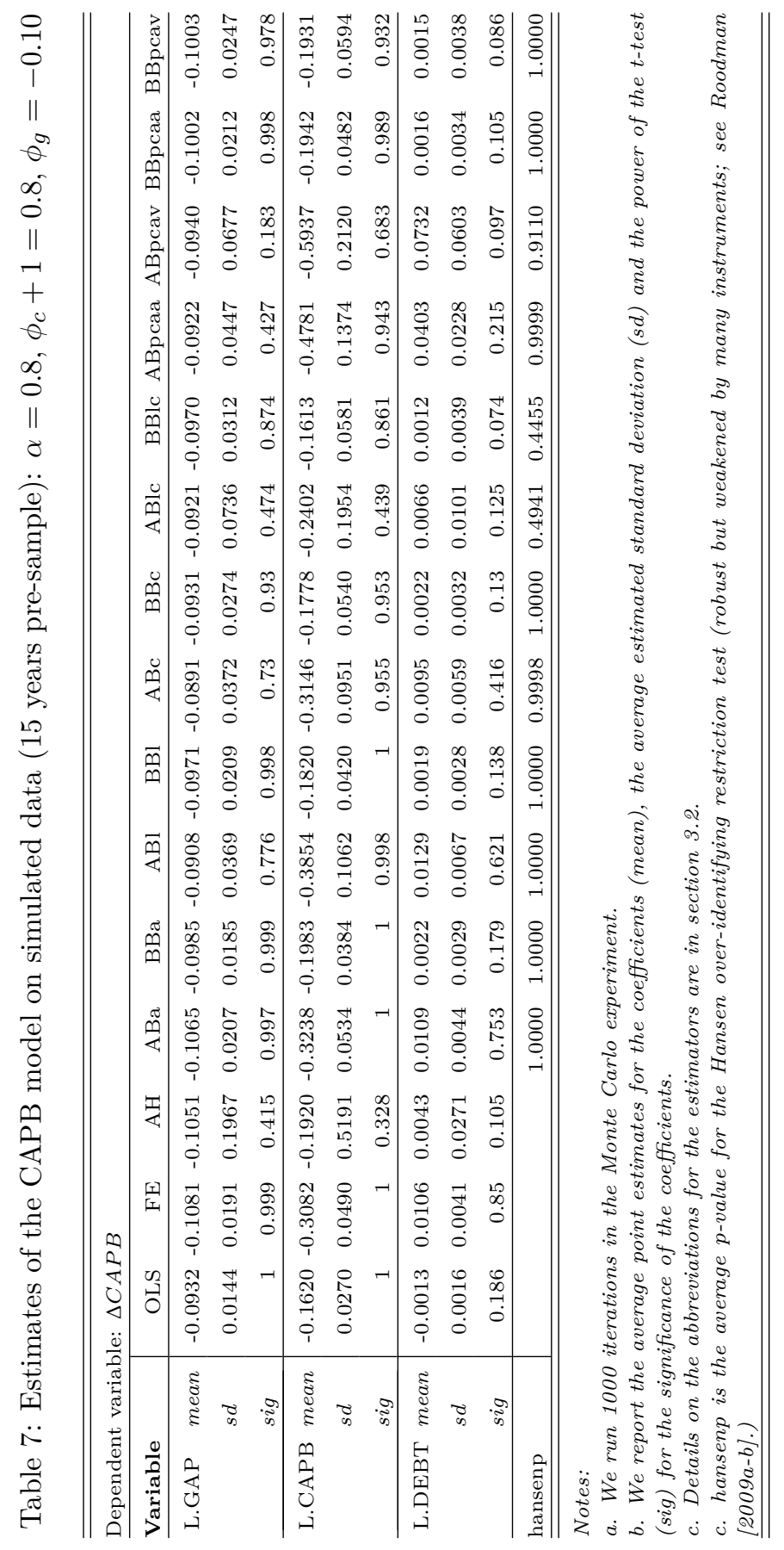




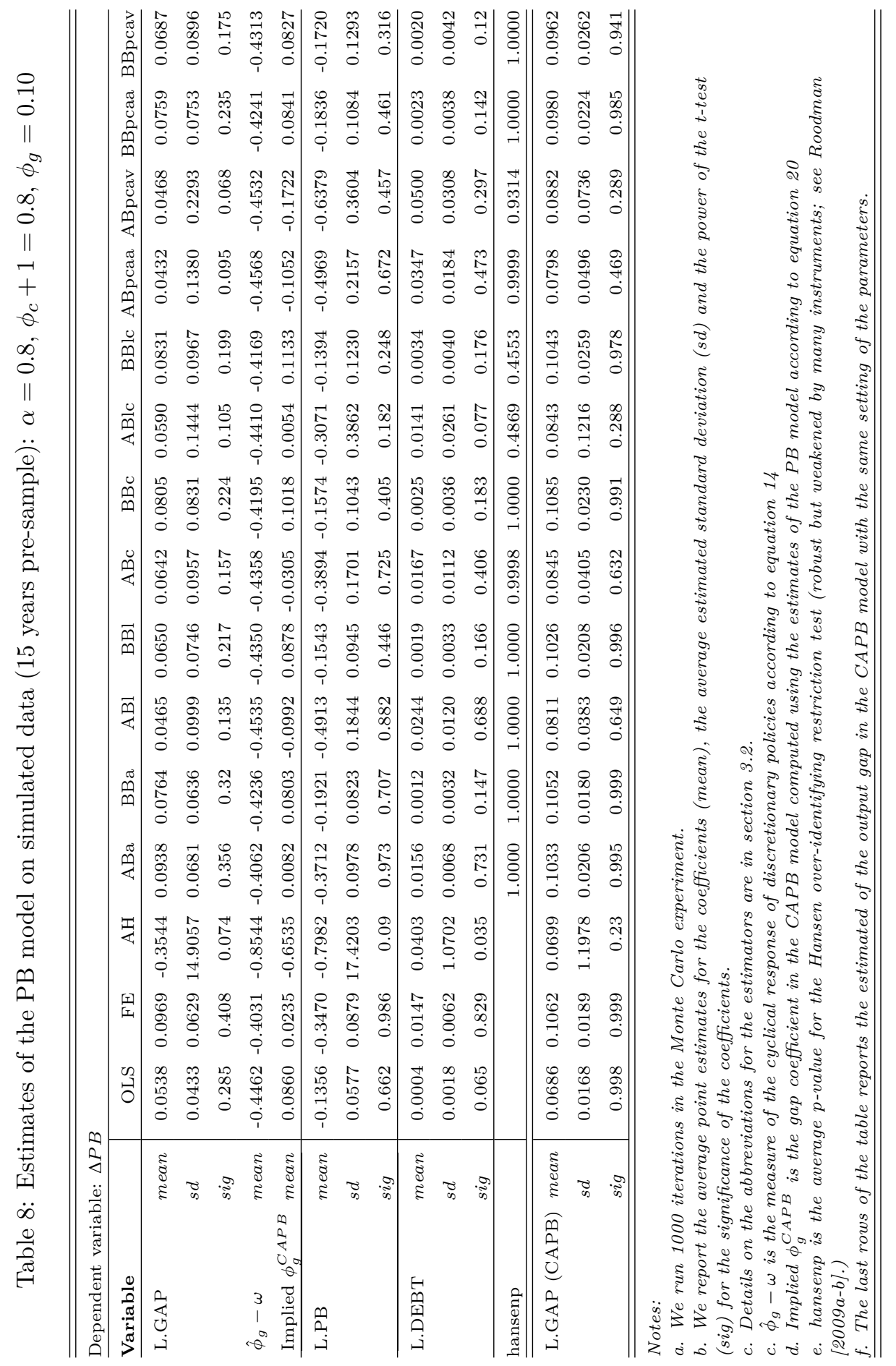




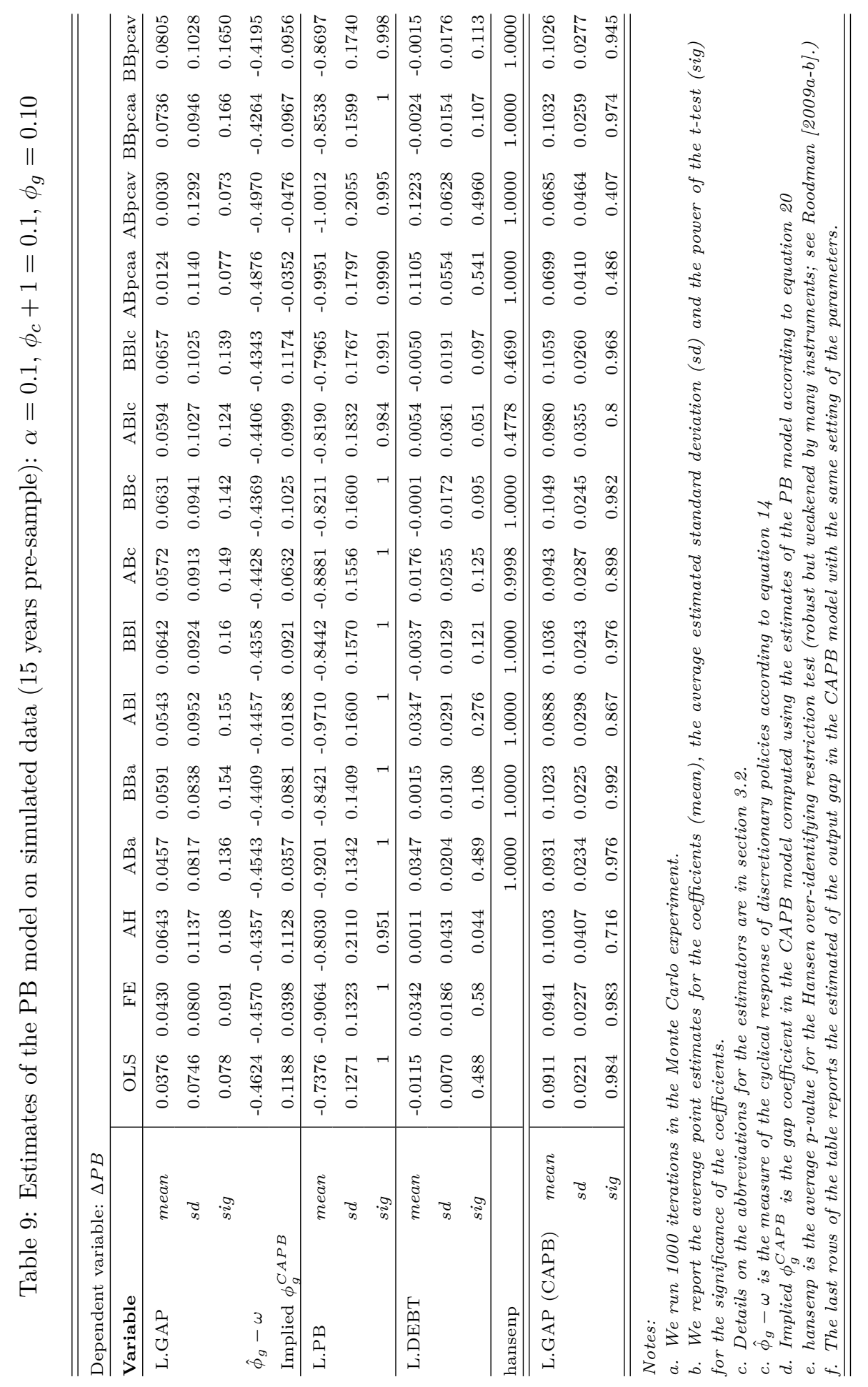




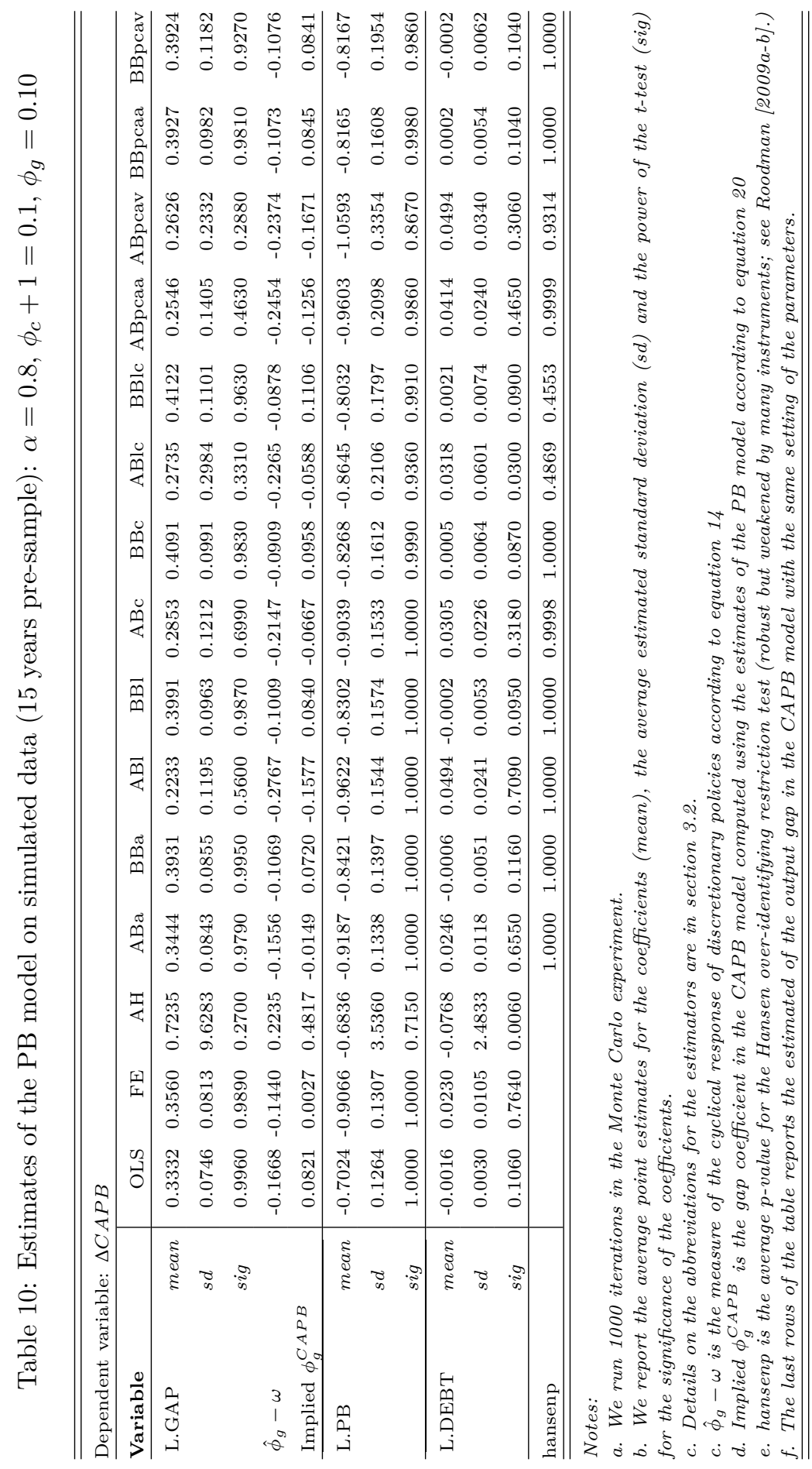




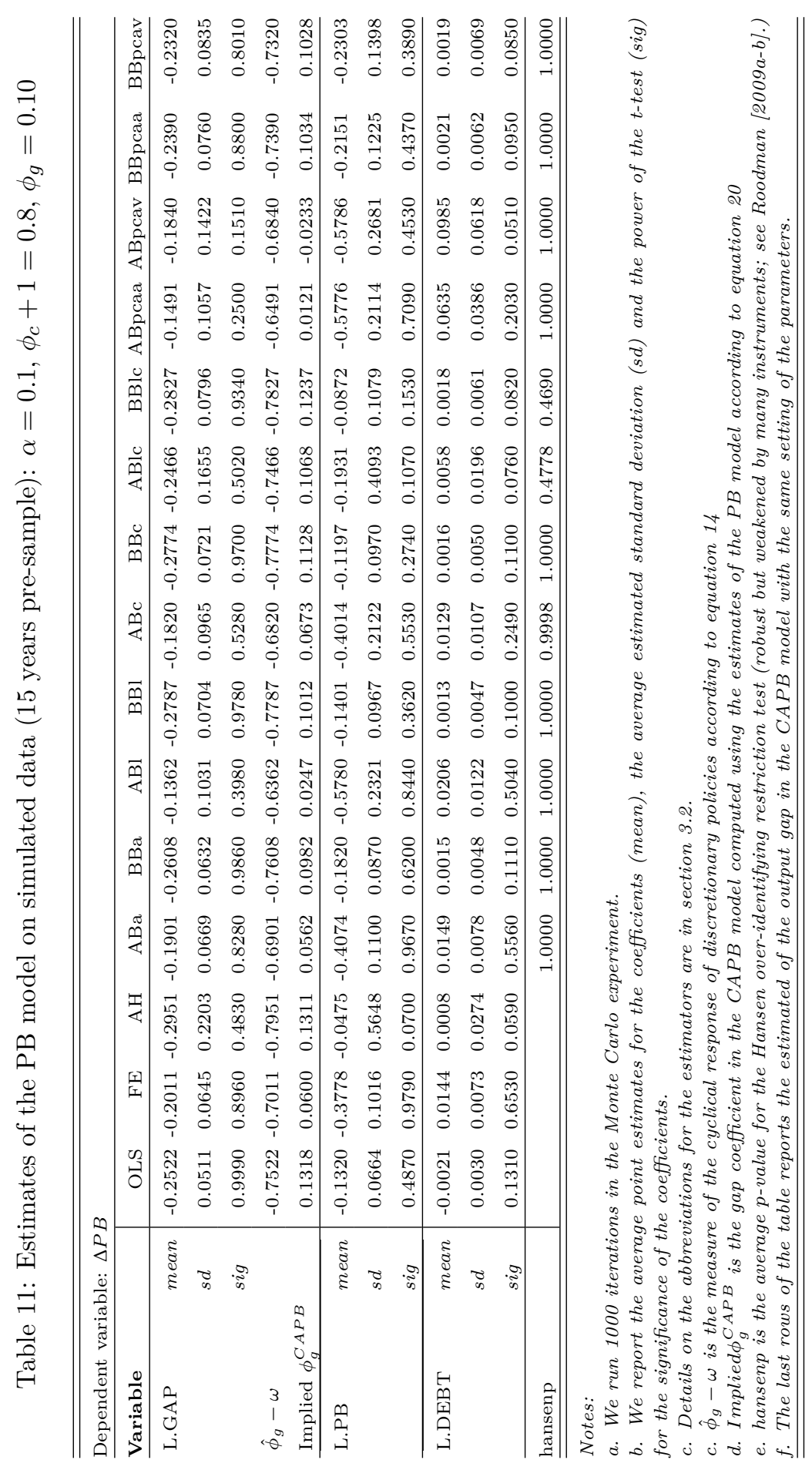


Table 12: CAPB model on real data: reproduction of Golinelli and Momigliano [2009]

\begin{tabular}{ll|rrrr}
\hline \hline \multicolumn{7}{l|}{ Dependent variable: $\triangle C A P B$} \\
\hline Variable & BBl1 orig. & BBl1 orig. rob. & BBl1 & & \\
\hline L.GAP & coeff & 0.069 & 0.069 & 0.069 & BB111 rob. \\
& $s d$ & 0.07 & 0.048 & 0.072 & 0.069 \\
& $t$ & 1 & 1.44 & 0.96 & 0.048 \\
\hline L.CAPB & coeff & -0.123 & -0.123 & -0.123 & -0.123 \\
& $s d$ & 0.073 & 0.104 & 0.076 & 0.104 \\
& $t$ & -1.67 & -1.18 & -1.62 & -1.18 \\
\hline L.DEBT & coeff & 0.004 & 0.004 & 0.004 & 0.004 \\
& $s d$ & 0.005 & 0.008 & 0.005 & 0.008 \\
& $t$ & 0.76 & 0.46 & 0.74 & 0.46 \\
\hline L.Maas & $c o e f f$ & -0.74 & -0.74 & -0.74 & -0.74 \\
& $s d$ & 0.176 & 0.212 & 0.182 & 0.212 \\
& $t$ & -4.21 & -3.49 & -4.07 & -3.49 \\
\hline Elect & $c o e f f$ & -0.466 & -0.466 & -0.466 & -0.466 \\
& $s d$ & 0.178 & 0.188 & 0.184 & 0.188 \\
& $t$ & -2.61 & -2.47 & -2.52 & -2.47 \\
\hline Obs. $(N)$ & $T)$ & 165 & 165 & 165 & 165 \\
\hline$T$ & 15 & 15 & 15 & 15 \\
Instruments & 124 & 124 & 124 & 124 \\
hansenp & & 1 & & 1 \\
Autocorrelation & 0.044 & 0.038 & 0.051 & 0.038 \\
\hline \hline
\end{tabular}

Notes:

a. Data are for 11 EMU Countries over the period 1994-2008. The dataset is the same used by Golinelli and Momigliano [2009].

b. We report the point estimates for the coefficients (coeff), the estimated standard deviation (sd) and the t-statistic (sig) for the significance of the coefficients.

c. Details on the abbreviations for the estimators are in section 3.2.

$d$. In the first column we reproduce the original estimates of the authors; in column 2 we make standard errors robust; in column 3 and 4 we replicate the estimates in column 1 and 2 with the most recent version of the estimation commands in Stata 12.

e. Instruments is the number of instruments used in the estimates.

f. hansenp is the p-value for the Hansen over-identifying restriction test (robust but weakened by many instruments; see Roodman [2009a-b].)

$g$. Autocorrelation reports the p-value of the residuals' second-order autocorrelation test. 


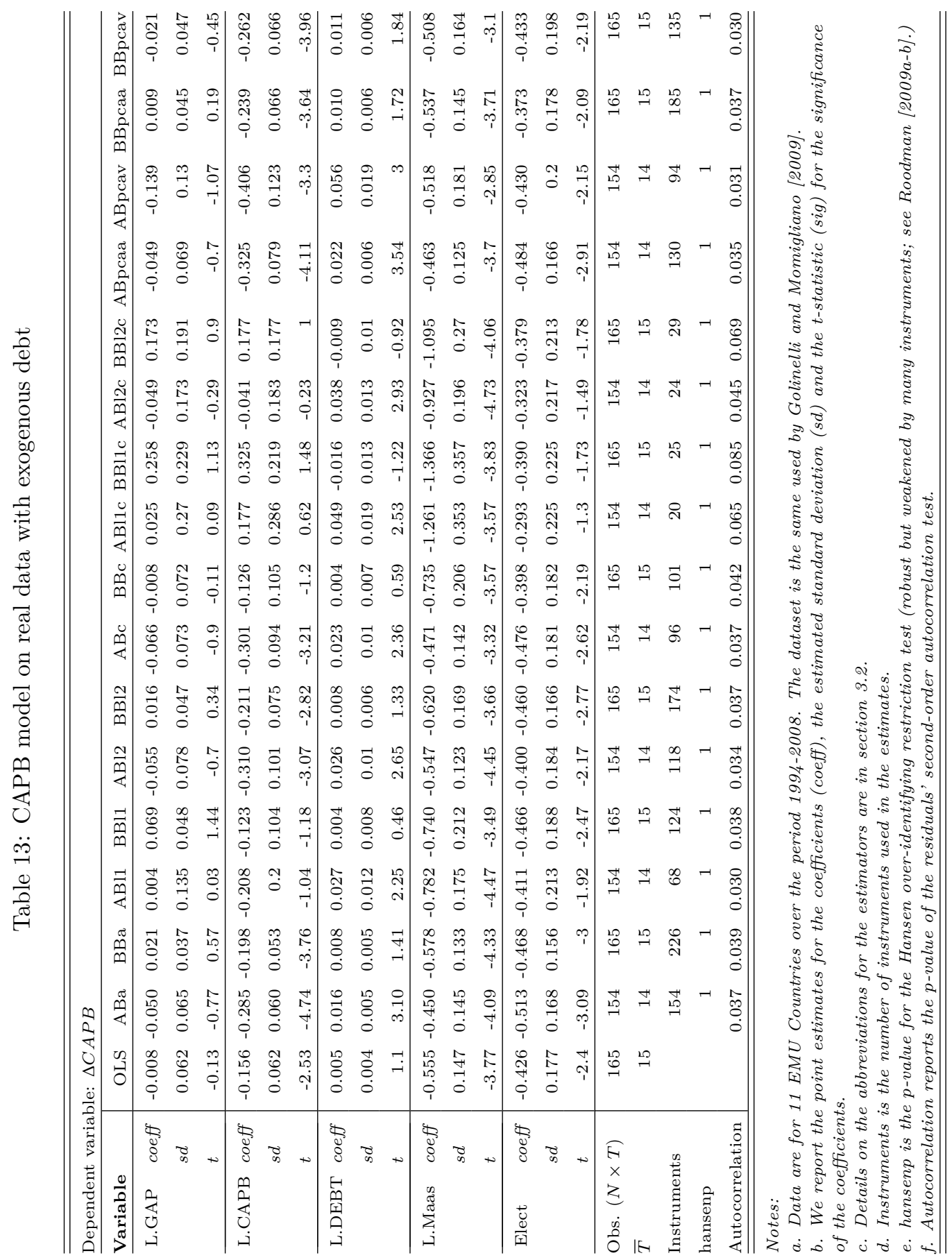




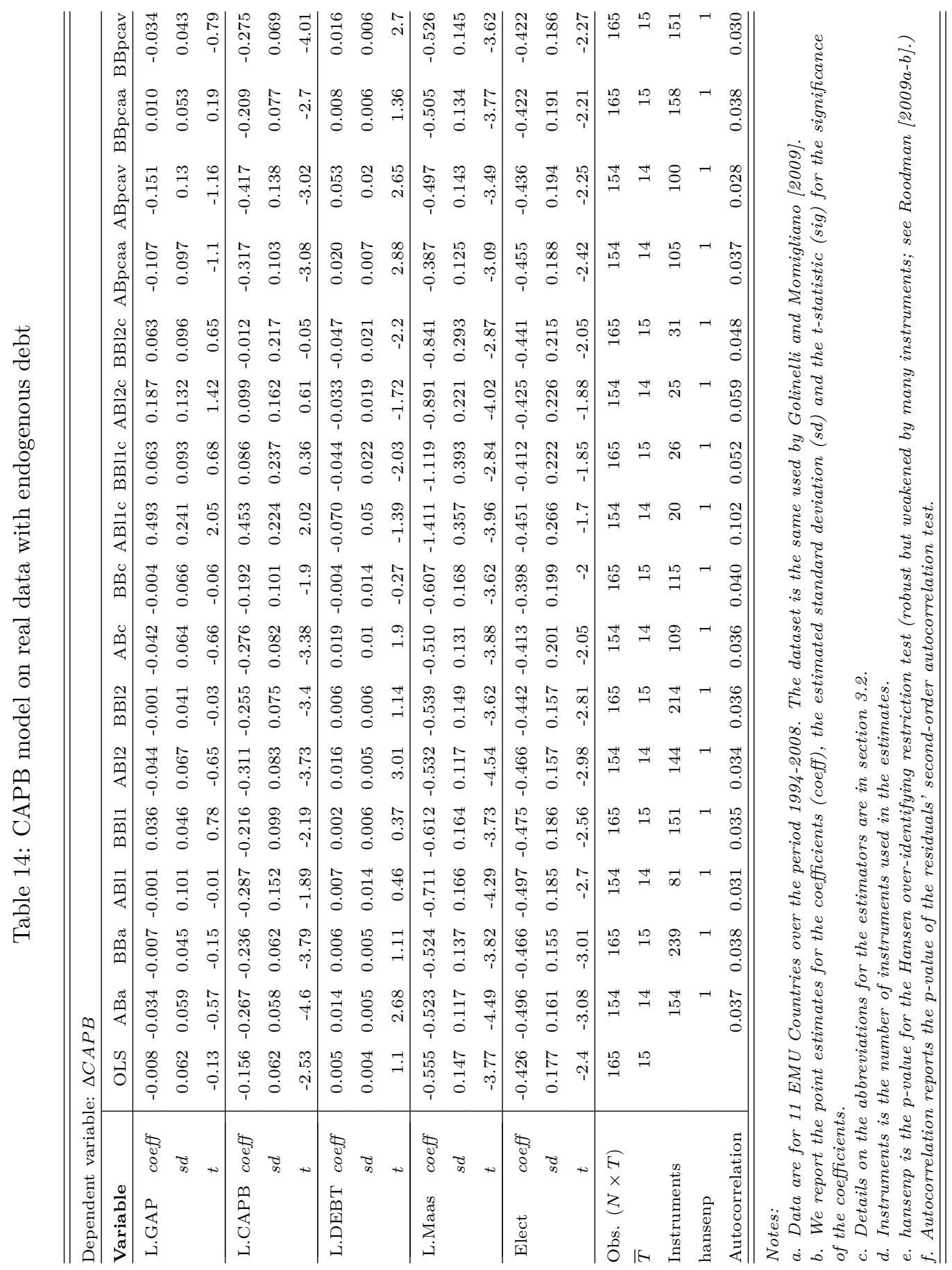




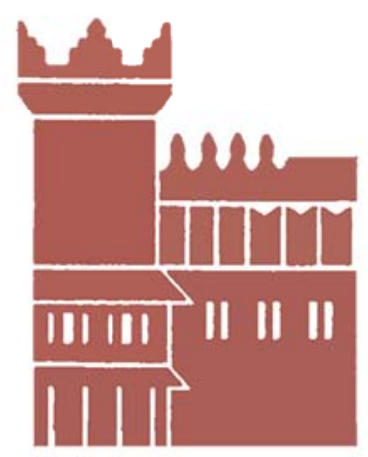

Alma Mater Studiorum - Università di Bologna DEPARTMENT OF ECONOMICS

Strada Maggiore 45

40125 Bologna - Italy

Tel. +39051 2092604

Fax +390512092664

http://www.dse.unibo.it 